\title{
Coupled reaction-diffusion and difference system of cell-cycle dynamics for hematopoiesis process with Dirichlet boundary conditions
}

\author{
Mostafa Adimy $^{\mathrm{a}}$, Abdennasser Chekroun $^{\mathrm{b}, *}$, Toshikazu Kuniya $^{\mathrm{c}}$ \\ ${ }^{a}$ Inria, Univ Lyon, Université Lyon 1, CNRS UMR 5208, Institut Camille Jordan, 43 \\ Bd. du 11 novembre 1918, F-69200 Villeurbanne Cedex, France. \\ ${ }^{b}$ Laboratoire d'Analyse Nonlinéaire et Mathématiques Appliquées, University of Tlemcen, \\ Tlemcen 13000, Algeria. \\ ${ }^{c}$ Graduate School of System Informatics, Kobe University, 1-1 Rokkodai-cho, Nada-ku, \\ Kobe 657-8501, Japan.
}

\begin{abstract}
In this paper, we propose a mathematical model describing the dynamics of hematopoietic stem cell (HSC) population during the cell-cycle. We take into account the spatial diffusion in the bone marrow. We consider for each cell, during its cell-cycle, which is a series of events that take place in the cell leading to its division, two main phases: quiescent phase and dividing phase. At the end of dividing phase, each cell divides and gives two daughter cells. A part of these daughter cells enters to the quiescent phase and the other part returns to the proliferating phase to divide again. Then, we obtain two nonlinear age-space-structured partial differential equations. Using the method of characteristics, we reduce this system to a coupled reaction-diffusion equation and difference one containing a nonlocal spatial term and a time delay. We start by studying the existence, uniqueness, positivity and boundedness of solutions for the reduced system. We then investigate the stability analysis and obtain a threshold condition for the global asymptotic stability of the trivial steady state by using a Lyapunov functional. We also obtain a sufficient condition for the existence and uniqueness of positive steady state
\end{abstract}

\footnotetext{
*Corresponding author

Email addresses: mostafa.adimy@inria.fr (Mostafa Adimy), chekroun@math.univ-lyon1.fr (Abdennasser Chekroun), tkuniya@port.kobe-u.ac.jp (Toshikazu Kuniya)
} 
by using the method of lower and upper solutions. Finally, we investigate the questions of persistence of the solution of the system when the trivial steady state is unstable.

Keywords: Age-space-structured PDE, Reaction-diffusion system with delay, Difference equation, Dirichlet boundary condition, Existence and uniqueness of positive steady state, Cell population dynamics 2010 MSC: 34B18, 35B35, 37N25, 92C37

\section{Introduction}

The cell-cycle is a series of tightly regulated molecular events controlling DNA replication and mitosis, producing two new daughter cells from a single parent cell [1]. Hematopoietic stem cells (HSCs) are undifferentiated cells that give rise to all types of blood lineage progenitors and the corresponding terminal differentiated blood cells: red blood cells, white blood cells, and platelets. This process is called hematopoiesis. The HSCs are located in the bone marrow before the mature blood cells enter the blood stream. They also have the ability to produce similar HSCs with the same maturity level (self-renewal). We consider that the cell-cycle is divided to two main phases: quiescent phase (or $\mathrm{G}_{0}$-phase) and dividing phase (or proliferating phase). Cells in the quiescent phase can stay their entire life $[2,3]$. However, in the proliferating phase cells are committed to undergo cell division a finite time later (this time was estimated between 1.4 and 4.3 days, [4, 2, 3]). Usually, $90 \%$ of HSCs are in the quiescent phase [5], while the remaining $10 \%$ are in the proliferating phase and committed to divide during mitosis at the end of this phase. After the division, each cell gives birth to two daughter cells which, either enter into the quiescent phase (long-term proliferation) or return to the proliferating phase (short-term proliferation) to divide again (see $[6,7,8])$.

To the best of our knowledge, the first mathematical model for HSC dynamics was introduced by Mackey [9] in 1978. Mackey's model is a system of two delay differential equations, to account for proliferating and quiescent HSC populations. This model has been analyzed in detail by $[9,10]$, and it has been applied to the study of hematopoietic dynamical disorders, characterized by significant oscillations of blood cell counts (see $[11,12,13,14,15,10,16,3,17]$ and the references therein). In all these works, only the long-term proliferation was taken into account, that is, all 
dividing cells at mitosis were assumed to enter directly to the quiescent phase. Yet, to be more realistic, one has to take also into account the short-term proliferation, in which a fraction of HSCs are always active in the cell-cycle and can induce a rapid proliferation of blood cells.

In [18], Adimy et al. studied a mathematical model for HSC dynamics taking into account the long- and short-term proliferation. They considered a system of delay differential-difference equations. They investigated the existence and stability of the two steady states; and the existence of a Hopf bifurcation for the positive steady state. Also see Diekmann, Getto and Nakata ([19]) for the situation that the cell-cycle incorporates a checkpoint in the sense that, at the passage of this point, a cell commits either to the division, the quiescence, or the apoptosis.

In many previous studies on mathematical models for HSC dynamics, the effects of spatial diffusion on the HSC population dynamics were neglected. However, it is believed that the diffusion of cells can play an important role in determining collective behavior of the cell population. It is known, for instance, that in many myeloproliferative disorders, the excessive proliferation of malignant HSCs can change normal spatial distribution of cell population in the bone marrow. In [20], we proposed and analyzed a mathematical model of HSC dynamics by taking into account the spacial diffusion of HSCs in the bone marrow (considered as a bounded domain with homogeneous Dirichlet boundary conditions). In this paper, however only the long-term proliferation was taken into account. In [21], a multi-scale model of erythropoiesis (the part of hematopoiesis that produces red blood cells) was introduced. It is based on ordinary differential equations (for intracellular network that regulates the behavior of each cell), reaction-diffusion system (for extracellular proteins as growth factors that act as signaling between cells) and individual based model (for the cell population). In [22], the authors described the evolution of leukemic HSCs in the bone marrow with a reaction-diffusion-convection system in porous medium. In another side, there have been extensive investigations on nonlocal and time-delayed population models in order to study the effects of spatial diffusion and time delay on the behavior of population (see for instance, [23] and [24]).

In [25], Adimy et al. proposed a mathematical model describing the dynamics of HSC population, taking into account an unbounded space. The model is a coupled reaction-diffusion equation and difference equation with delay. They studied the existence of traveling wave fronts connecting the zero steady state with the unique positive uniform one. They used a monotone 
iteration technique coupled with the upper and lower solutions method.

In this paper, as in $[25,18]$, we consider the long- and short-term proliferation of HSC population, that is, a proportion of dividing daughter cells enters into the quiescent phase, while the other proportion returns to the proliferating phase to divide again. As in [25], we take into account the spatial distribution of HSC population in the bone marrow. However, we consider a bounded space with Dirichlet boundary conditions. The model is formulated as a nonlinear system of two age-structured partial differential equations with diffusion. Using the method of characteristics, we reduce it to a coupled system of reaction-diffusion equation and difference equation with a nonlocal spatial term and time delay. For the reduced system, we investigate the existence, uniqueness, positivity and boundedness of solutions. By constructing a Lyapunov function, we obtain a condition for the global asymptotic stability of the trivial steady state. We also obtain a sufficient condition for the existence and uniqueness of positive steady state by using the method of lower and upper solutions. Finally, we prove that when the trivial steady state is unstable, we still have the persistence (see [26]) of the solution of the system.

The organization of the paper is as follows. In Section 2, we construct our model and describe the biological background. In Section 3, we reduce the model to a coupled system of reaction-diffusion equation and difference equation with a nonlocal spatial term and time delay. In Section 4, we obtain a condition for the existence and uniqueness of positive solutions. In Section 5, we obtain a threshold condition which determines either the global asymptotic stability or instability of the trivial steady state. The proof is done by constructing a Lyapunov function. In Section 6, we obtain sufficient conditions for the existence and uniqueness of positive steady state by constructing upper and lower solutions. In Section 7, we study the questions of weak persistence and persistence of the solution of the system when the trivial steady state is unstable. The Section 8 ends the paper and summarizes the results obtained.

\section{A model for HSC dynamics (age-space-structured PDE)}

We denote by $p(t, x, a)$ and $n(t, x, a)$ the densities of proliferating and non-proliferating (quiescent) hematopoietic stem cell (HSC) population, respectively. The variable $t \geq 0$ represents the time, $a$ is the time (age) spent by each cell in a phase (proliferating or quiescent) and $x \in \Omega$ is the position. 
For the proliferating cells, the age varies between 0 and $\tau$ and for the quiescent cells from 0 to $+\infty$. We set $d_{1} \geq 0$ and $d_{2} \geq 0$ the rates of diffusion of cells in each compartment. For a more clear understanding of the model, we give in Figure 1 a schematic representation of this process.

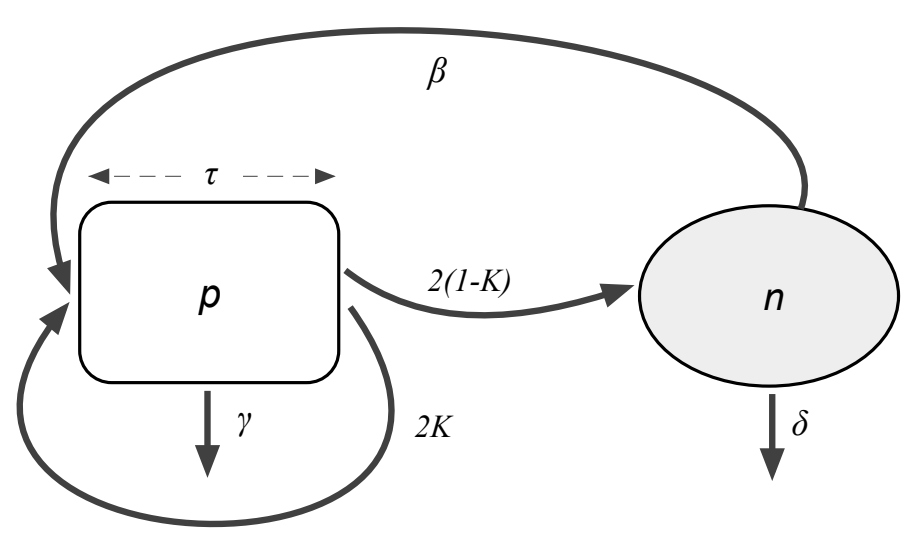

Figure 1: A schematic representation of the cell-cycle.

In the proliferating phase, cells can be lost by apoptosis (programmed cell death) at a rate $\gamma \geq 0$. At the end of this phase (that is when cells have spent a time $a=\tau$ ), each cell divides in two daughter cells. A part $(K \in[0,1])$ of these daughter cells returns immediately to the proliferating phase to go over a new cell-division cycle while the other part $(1-K)$ enters directly the quiescent phase. Quiescent cells can either be lost randomly at a rate $\delta \geq 0$, which takes into account the cellular differentiation, or enter into the proliferating phase at a rate $\beta \geq 0$. Set

$$
N(t, x)=\int_{0}^{+\infty} n(t, x, a) \mathrm{d} a, \quad x \in \Omega, \quad t \geq 0 .
$$

We assume that the introduction rate $\beta:=\beta(N(t, x))$ depends on the total population $N(t, x)$ of quiescent cells (see [9]) and it is a continuously differentiable and strictly decreasing function with $\lim _{N \rightarrow+\infty} \beta(N)=0$. Typically, $\beta$ is a Hill function (see $[9,3,17]$ ) given by

$$
\beta(N)=\frac{\beta_{0} \theta^{r}}{\theta^{r}+N^{r}}, \quad r>1 .
$$




\begin{tabular}{|c|c|}
\hline Parameters & Interpretation (units) \\
\hline$\delta$ & Quiescent cells mortality rate $\left(\right.$ day $\left.^{-1}\right)$ \\
\hline$\gamma$ & Apoptosis rate $\left(\right.$ day $\left.^{-1}\right)$ \\
\hline$\beta_{0}$ & Maximum introduction rate $\left(\right.$ day $\left.^{-1}\right)$ \\
\hline$r$ & Sensitivity of the introduction rate (none) \\
\hline$\theta$ & $\theta$ is such that $\beta(\theta)=\beta_{0} / 2\left(\right.$ cells. $\left.\mathrm{kg}^{-1}\right)$ \\
\hline$\tau$ & Duration of proliferating phase (days) \\
\hline$K$ & Short proliferating rate $\left(\right.$ day $\left.^{-1}\right)$ \\
\hline$d_{1}$ & Diffusion rate of resting cells $\left(\right.$ day $\left.^{-1}\right)$ \\
\hline$d_{2}$ & Diffusion rate of proliferating cells $\left(\right.$ day $\left.^{-1}\right)$ \\
\hline
\end{tabular}

Table 1: Model parameters with their interpretation.

All the parameters cited before are described in the Table 1.

At the end of the proliferating phase $(a=\tau)$, a part, $1-K$, of daughter cells enters to the quiescent phase. Then, we have the following boundary condition

$$
n(t, x, 0)=2(1-K) p(t, x, \tau) .
$$

The other part, $K$, returns to the proliferating phase to divide again. So, the new cells entering the proliferation phase are given by the following expression

$$
\begin{aligned}
p(t, x, 0) & =\int_{0}^{+\infty} \beta(N(t, x)) n(t, x, a) d a+2 K p(t, x, \tau), \\
& =\beta(N(t, x)) N(t, x)+2 K p(t, x, \tau) .
\end{aligned}
$$

We consider one-dimensional space, $\Omega:=\left(l_{1}, l_{2}\right)$. Then, we describe the evolution of the HSC population by the following partial differential system, for $t>0$ and $x \in\left(l_{1}, l_{2}\right)$,

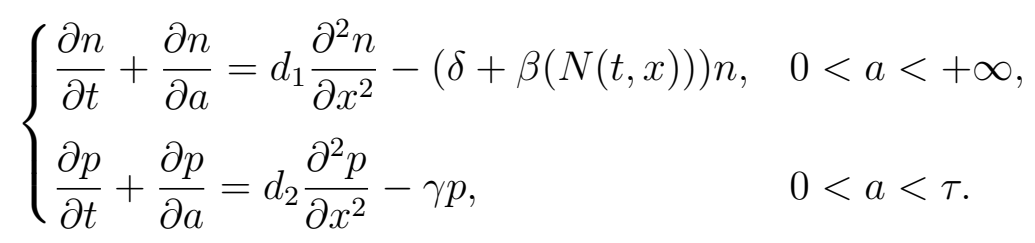

This system is completed by a Dirichlet boundary conditions

$$
\begin{cases}n(t, x, a)=0, & x \in \partial \Omega, \quad 0<a<+\infty \\ p(t, x, a)=0, & x \in \partial \Omega, \quad 0<a<\tau,\end{cases}
$$


and initial conditions

$$
\begin{cases}n(0, x, a)=n_{0}(x, a), & x \in \Omega, \quad 0<a<+\infty, \\ p(0, x, a)=p_{0}(x, a), & x \in \Omega, \quad 0<a<\tau .\end{cases}
$$

We also add the following natural hypothesis

$$
\lim _{a \rightarrow+\infty} n(t, x, a)=0
$$

Without loss of generality, we use the following change of variable

$$
x \longmapsto \frac{\pi\left(x-l_{1}\right)}{l_{2}-l_{1}} .
$$

The domain is transformed to $\Omega=(0, \pi)$ and the diffusion coefficients become $d_{1}\left(\pi /\left(l_{2}-l_{1}\right)\right)^{2}$ and $d_{2}\left(\pi /\left(l_{2}-l_{1}\right)\right)^{2}$ (denoted again by $d_{1}$ and $\left.d_{2}\right)$. In this case, we get the following boundary condition, for $t>0$,

$$
\begin{cases}n(t, 0, a)=n(t, \pi, a)=0, & 0<a<+\infty \\ p(t, 0, a)=p(t, \pi, a)=0, & 0<a<\tau\end{cases}
$$

\section{Reduction of the system (reaction-diffusion and difference sys- tem)}

We integrate the first equation of (3) and we obtain

$$
\frac{\partial N}{\partial t}(t, x)+n(t, x,+\infty)-n(t, x, 0)=d_{1} \frac{\partial^{2} N}{\partial x^{2}}(t, x)-(\delta+\beta(N(t, x))) N(t, x) .
$$

By using the equality (1) and the fact that $\lim _{a \rightarrow+\infty} n(t, x, a)=0$, the equation (4) becomes

$$
\frac{\partial N}{\partial t}(t, x)=d_{1} \frac{\partial^{2} N}{\partial x^{2}}(t, x)-(\delta+\beta(N(t, x))) N(t, x)+2(1-K) p(t, x, \tau) .
$$

By using the characteristics method (see [25, 27, 28]), for the second equation of (3), we get for $t>\tau$,

$$
p(t, x, \tau)=\int_{0}^{\pi} p(t-\tau, y, 0)\left[\frac{2}{\pi} \sum_{k=1}^{+\infty} \sin (k x) \sin (k y) e^{-\left(d_{2} k^{2}+\gamma\right) \tau}\right] d y
$$


By putting $u(t, x)=p(t, x, 0)$ and using (2), we get

$$
u(t, x)=\beta(N(t, x)) N(t, x)+2 K \int_{0}^{\pi} u(t-\tau, y)\left[\frac{2}{\pi} \sum_{k=1}^{+\infty} \sin (k x) \sin (k y) e^{-\left(d_{2} k^{2}+\gamma\right) \tau}\right] d y .
$$

Likewise, the equation (5) becomes, for $t>\tau$,

$$
\begin{aligned}
\frac{\partial N}{\partial t}(t, x)= & d_{1} \frac{\partial^{2} N}{\partial x^{2}}(t, x)-(\delta+\beta(N(t, x))) N(t, x) \\
& +2(1-K) \int_{0}^{\pi} u(t-\tau, y) \frac{2}{\pi} \sum_{k=1}^{+\infty} \sin (k x) \sin (k y) e^{-\left(d_{2} k^{2}+\gamma\right) \tau} d y .
\end{aligned}
$$

We use the translation $t \mapsto t-\tau$ to make the last equations defined for $t>0$ instead of $t>\tau$. Furthermore, by using the characteristics method for the case $t \leq \tau$, the initial conditions are defined on $[-\tau, 0]$. We put, for $i=1,2$,

$$
\Gamma_{i}(\tau, x, y)=\frac{2}{\pi} \sum_{k \geq 1} e^{-k^{2} d_{i} \tau} \sin (k x) \sin (k y), \quad x, y \in[0, \pi]
$$

The function $\Gamma_{i}$ satisfies the estimation (the proof can be found in [29])

$$
0<\Gamma_{i}(\tau, x, y)<\frac{1}{\pi}\left(\frac{2}{e^{d_{i} \tau}-1}\right), \quad \text { for } \quad x, y \in(0, \pi) .
$$

Then, the system (3) can be reduced to the following reaction-diffusion and difference system, for $t>0$ and $x \in[0, \pi]$,

$$
\left\{\begin{array}{l}
\frac{\partial N}{d t}(t, x)=d_{1} \frac{\partial^{2} N}{\partial x^{2}}(t, x)-(\delta+\beta(N(t, x))) N(t, x) \\
\quad+2(1-K) e^{-\gamma \tau} \int_{0}^{\pi} \Gamma_{2}(\tau, x, y) u(t-\tau, y) d y \\
u(t, x)=\beta(N(t, x)) N(t, x)+2 K e^{-\gamma \tau} \int_{0}^{\pi} \Gamma_{2}(\tau, x, y) u(t-\tau, y) d y \\
N(t, 0)=N(t, \pi)=u(t, 0)=u(t, \pi)=0 \\
N(0, x)=N_{0}(x), \\
u(\theta, x)=u_{0}(\theta, x), \quad \theta \in[-\tau, 0]
\end{array}\right.
$$

In all the sequel, we concentrate our study on the reduced system (6). 


\section{Existence, uniqueness and positivity of solutions}

In this section, we focus on the existence and uniqueness of solutions of the system (6) and we establish other properties such that the positivity and a principle of comparison. The existence result is obtained by constructing two monotone sequences of bounded lower and upper solutions, which converge to the unique solution of (6). We start by establishing the uniqueness and positivity of solutions $(N(t, x), u(t, x))$ of the system (6).

Let $X=C([0, \pi], \mathbb{R})$ be the Banach space of all continuous functions from $[0, \pi]$ to $\mathbb{R}$ with the usual supremum norm $|\cdot|_{X}$ and $X^{+}:=\{\phi \in X: \phi(x) \geq$ 0 , for all $x \in[0, \pi]\}$. We put, for $i=1,2$ and $v \in X$,

$$
\left(T_{i}(t) v\right)(x):=\int_{0}^{\pi} \Gamma_{i}(t, x, y) v(y) \mathrm{d} y, \quad t>0, x \in[0, \pi] .
$$

$\left(T_{i}(t)\right)_{t \geq 0}$ is a strongly continuous semigroup of linear operators on $X$. Consider also the nonlinear functions $f, g: X \rightarrow X$ defined, for $v \in X$, by

$$
f(v)(x)=(\delta+\beta(v(x))) v(x) \quad \text { and } \quad g(v)(x)=\beta(v(x)) v(x), \quad x \in[0, \pi] .
$$

Let $C:=C([-\tau, 0], X)$ be the Banach space of continuous functions from $[-\tau, 0]$ into $X$ with the classical supremum norm $\|\cdot\|_{C}$ and the closed cone $C^{+}:=C\left([-\tau, 0], X^{+}\right)$. We identify the functions $N, u:[0,+\infty) \times[0, \pi] \rightarrow \mathbb{R}$, (respectively, $u_{0}:[-\tau, 0] \times[0, \pi] \rightarrow \mathbb{R}$ ) as functions from $[0,+\infty$ ) (respectively, $[-\tau, 0])$ into $X$, by putting $N(t)(x)=N(t, x)$ and $u(t)(x)=u(t, x)$ (respectively, $u_{0}(\theta)(x)=u_{0}(\theta, x)$ ). Since the function $\beta$ is continuously differentiable, then the functions $f$ and $g$ are also continuously differentiable on $X$ and satisfy $f\left(X^{+}\right) \subseteq X^{+}, g\left(X^{+}\right) \subseteq X^{+}$. The system (6) reads, for $t>0$,

$$
\begin{cases}\frac{\mathrm{d}}{\mathrm{d} t} N(t) & =d_{1} \Delta N(t)-f(N(t))+2(1-K) e^{-\gamma \tau} T_{2}(\tau) u(t-\tau), \\ u(t) & =g(N(t))+2 K e^{-\gamma \tau} T_{2}(\tau) u(t-\tau)\end{cases}
$$

where $N(t)(0)=N(t)(\pi)=u(t)(0)=u(t)(\pi)=0$ with initial conditions

$$
N(0)=N_{0} \in X^{+} \quad \text { and } \quad u(\theta)=u_{0}(\theta), \theta \in[-\tau, 0], \quad u_{0} \in C^{+} .
$$

An abstract integral formulation of the system (7) (or equivalently (6)) (in 
terms of $\left.T_{1}(t)\right)$ is

$$
\left\{\begin{aligned}
N(t)= & T_{1}(t) N_{0}-\int_{0}^{t} T_{1}(t-s) f(N(s)) \mathrm{d} s \\
& +2(1-K) e^{-\gamma \tau} \int_{0}^{t} T_{1}(t-s) T_{2}(\tau) u(s-\tau) \mathrm{d} s \\
u(t)= & g(N(t))+2 K e^{-\gamma \tau} T_{2}(\tau) u(t-\tau) .
\end{aligned}\right.
$$

The solutions of (8) are called mild solutions of (7). If the solution of (8) is classical, then the systems (7) and (8) are equivalent.

We will concentrate now on the existence, uniqueness, positivity and boundedness of the solutions of the system (8). The next result proves the uniqueness of bounded solutions.

Proposition 1. For each initial condition $\left(N_{0}, u_{0}\right) \in X \times C$, the system (8) has at most one solution.

Proof. Let consider $\left(N^{1}, u^{1}\right)$ and $\left(N^{2}, u^{2}\right)$ two bounded solutions of (8) defined on $[0,+\infty)$, with the same initial condition $\left(N_{0}, u_{0}\right) \in X \times C$. We put $N=N^{1}-N^{2}$ and $u=u^{1}-u^{2}$. Then, (8) gives

$$
\left\{\begin{aligned}
N(t)= & -\int_{0}^{t} T_{1}(t-s)\left(f\left(N^{1}(s)\right)-f\left(N^{2}(s)\right)\right) \mathrm{d} s \\
& +2(1-K) e^{-\gamma \tau} \int_{0}^{t} T_{1}(t-s) T_{2}(\tau) u(s-\tau) \mathrm{d} s \\
u(t)= & g\left(N^{1}(t)\right)-g\left(N^{2}(t)\right)+2 K e^{-\gamma \tau} T_{2}(\tau) u(t-\tau) .
\end{aligned}\right.
$$

We decompose the interval $[0,+\infty)$ into $[0, \tau] \cup[\tau, 2 \tau] \cup \ldots \cup[n \tau,(n+1) \tau] \cup \ldots$, and we proceed by steps in each subinterval. Let $t \in[0, \tau]$. Then, $u(t-\tau)=0$ and we have

$$
\left\{\begin{array}{l}
N(t)=-\int_{0}^{t} T_{1}(t-s)\left(f\left(N^{1}(s)\right)-f\left(N^{2}(s)\right)\right) \mathrm{d} s \\
u(t)=g\left(N^{1}(t)\right)-g\left(N^{2}(t)\right) .
\end{array}\right.
$$

As $N^{1}$ and $N^{2}$ are continuous and bounded, there exists $B>0$ such that $\left|N^{1}(s)\right|_{X},\left|N^{2}(s)\right|_{X}<B$, for all $s \in[0, \tau]$. Since the function $\beta$ is continuously differentiable, there exists $L_{B}>0$ such that

$$
\left\{\begin{array}{l}
\left|f\left(N^{1}(s)\right)-f\left(N^{2}(s)\right)\right|_{X} \leq L_{B}\left|N^{1}(s)-N^{2}(s)\right|_{X}=L_{B}|N(s)|_{X}, \\
\left|g\left(N^{1}(s)\right)-g\left(N^{2}(s)\right)\right|_{X} \leq L_{B}\left|N^{1}(s)-N^{2}(s)\right|_{X}=L_{B}|N(s)|_{X} .
\end{array}\right.
$$


Furthermore, for $s>0$ and $x, y \in(0, \pi)$,

$$
\begin{aligned}
& \int_{0}^{\pi} \Gamma_{1}(s, x, y) \mathrm{d} y=\frac{2}{\pi} \sum_{k=1}^{+\infty} \sin (k x)\left(\int_{0}^{\pi} \sin (k y) \mathrm{d} y\right) e^{-k^{2} d_{1} s}, \\
& =\frac{2}{\pi} \sum_{k=1}^{+\infty} \sin (k x)\left[-\frac{\cos (k y)}{k}\right]_{0}^{\pi} e^{-k^{2} d_{1} s}=\frac{4}{\pi} \sum_{k=1}^{+\infty} \frac{\sin (2 k-1) x}{2 k-1} e^{-(2 k-1)^{2} d_{1} s} .
\end{aligned}
$$

Let

$$
C:=\max _{(s, x) \in[0, \tau] \times[0, \pi]} \frac{4}{\pi} \sum_{k=1}^{+\infty} \frac{\sin (2 k-1) x}{2 k-1} e^{-(2 k-1)^{2} d_{1} s} \in(0,+\infty) .
$$

Then, the first equation of (9) implies

$$
|N(t)|_{X} \leq L_{B} C \int_{0}^{t}|N(s)|_{X} \mathrm{~d} s, \quad t \in[0, \tau] .
$$

By Gronwall's lemma, we conclude that $N(t)(x)=0$, for all $t \in[0, \tau]$ and $x \in[0, \pi]$. The second equation of (9) implies that $u(t)(x)=0$, for all $t \in[0, \tau]$ and $x \in[0, \pi]$. Applying the same argument in each interval $[k \tau,(k+1) \tau], k \in \mathbb{N}^{*}$, we conclude that $N(t)=u(t)=0$, for all $t \in[0,+\infty)$. This completes the proof.

The next proposition proves the positivity of bounded solutions of the system (7).

Proposition 2. Let $\left(N_{0}, u_{0}\right) \in X^{+} \times C^{+}$and $(N, u)$ be a solution of (7) corresponding to the initial condition $\left(N_{0}, u_{0}\right)$. Then, $(N, u)$ is nonnegative.

Proof. We put $P(t)=e^{-\rho t} N(t)$, for $\rho>0$. Then, the first equation of $(7)$ implies

$\frac{\mathrm{d}}{\mathrm{d} t} P(t)=d_{1} \Delta P(t)-(\rho+\delta+\beta(N(t))) P(t)+2(1-K) e^{-\rho t} e^{-\gamma \tau} T_{2}(\tau) u(t-\tau)$.

We use an iterative method on each interval $[k \tau,(k+1) \tau], k \in \mathbb{N}$. Let $t \in[0, \tau]$. As $t-\tau \in[-\tau, 0]$, then $u(t-\tau)=u_{0}(t-\tau) \geq 0$. Consequently,

$$
\frac{\mathrm{d}}{\mathrm{d} t} P(t) \geq d_{1} \Delta P(t)-(\rho+\delta+\beta(N(t))) P(t) .
$$


We put $l_{\rho}(t)=\rho+\delta+\beta(N(t))$, for $t \in[0, \tau]$. Due to the fact that $N$ is bounded, we can take $\rho>0$ large enough to ensure that $l_{\rho}(t) \geq 0$, for all $t \in[0, \tau]$. Then,

$$
\left\{\begin{array}{l}
\frac{\mathrm{d}}{\mathrm{d} t} P(t)-d_{1} \Delta P(t)+l_{\rho}(t) P(t) \geq 0 \\
P(0)=N_{0} \geq 0
\end{array}\right.
$$

As $l_{\rho}(t) \geq 0$, by the maximum principle (see [30]), we get $P(t) \geq 0$ for $t \in[0, \tau]$. That is to say that $N(t) \geq 0$, for all $t \in[0, \tau]$. Moreover, the second equation of $(7)$ implies that $u(t) \geq 0$, for all $t \in[0, \tau]$. We repeat the same argument for $t \in[k \tau,(k+1) \tau]$, with $k=1,2, \ldots$ Then, we obtain the positivity of the solutions of $(7)$.

Now, we return to the existence of solutions of (7). We need to define the notion of upper and lower solutions of (7).

Definition 4.1. A pair of functions $(\bar{N}, \bar{u})$ (respectively, $(\underline{N}, \underline{u})$ ), is called upper (respectively, lower) solution of $(7)$ on $[0,+\infty)$, if $\bar{N}$ (respectively, $\underline{N}$ ) is $C^{1}$ in $t \in(0,+\infty), C^{2}$ in $x \in[0, \pi], \bar{u}$ (respectively, $\underline{u}$ ) is in $C([-\tau,+\infty), X)$, null at $x=0, \pi$, and they satisfy the following inequalities

$$
\begin{cases}\frac{\mathrm{d}}{\mathrm{d} t}(t) & \geq d_{1} \Delta \bar{N}(t)-f(\bar{N}(t))+2(1-K) e^{-\gamma \tau} T_{2}(\tau) \bar{u}(t-\tau) \\ \bar{u}(t) & \geq \beta(\underline{N}(t)) \bar{N}(t)+2 K e^{-\gamma \tau} T_{2}(\tau) \bar{u}(t-\tau)\end{cases}
$$

and

$$
\left\{\begin{array}{l}
\frac{\mathrm{d}}{\mathrm{d} t} \underline{N}(t) \leq d_{1} \Delta \underline{N}(t)-f(\underline{N}(t))+2(1-K) e^{-\gamma \tau} T_{2}(\tau) \underline{u}(t-\tau), \\
\underline{u}(t) \leq \beta(\bar{N}(t)) \underline{N}(t)+2 K e^{-\gamma \tau} T_{2}(\tau) \underline{u}(t-\tau) .
\end{array}\right.
$$

A nonnegative bounded upper (respectively, lower) solution $(\bar{N}, \bar{u})$ (respectively, $(\underline{N}, \underline{u}))$ of $(7)$ satisfies the following proposition.

Proposition 3. Let $(\bar{N}, \bar{u})$ (respectively, $(\underline{N}, \underline{u})$ ) be a nonnegative upper (respectively, lower) solution of $(7)$ on $[0,+\infty)$, such that $\underline{N}(0) \leq \bar{N}(0)$ and $\underline{u}(\theta) \leq \bar{u}(\theta)$, for all $-\tau \leq \theta \leq 0$. Then, $\underline{N}(t) \leq \bar{N}(t)$ and $\underline{u}(t) \leq \bar{u}(t)$, for all $t \in[0,+\infty)$. 
Proof. We put

$N=\bar{N}-\underline{N}$, on $[0,+\infty) \times[0, \pi]$ and $u=\bar{u}-\underline{u}$, on $[-\tau,+\infty) \times[0, \pi]$.

We have the following inequalities

$\left\{\begin{array}{l}\frac{\mathrm{d}}{\mathrm{d} t} N(t)-d_{1} \Delta N(t)+f(\bar{N}(t))-f(\underline{N}(t)) \geq 2(1-K) e^{-\gamma \tau} T_{2}(\tau) u(t-\tau), \\ u(t) \geq \beta(\underline{N}(t)) \bar{N}(t)-\beta(\bar{N}(t)) \underline{N}(t)+2 K e^{-\gamma \tau} T_{2}(\tau) u(t-\tau),\end{array}\right.$

with $N(0)=\bar{N}(0)-\underline{N}(0) \geq 0$ and $u(t)=\bar{u}(t)-\underline{u}(t) \geq 0$, for $t \in[-\tau, 0]$. We use the same iterative idea as in the proof of Proposition 2. By steps, let $t \in[0, \tau]$. Then, $t-\tau \in[-\tau, 0]$ and $u(t-\tau)=\bar{u}(t-\tau)-\underline{u}(t-\tau) \geq 0$. Thus, we obtain for $t \in[0, \tau]$,

$$
\left\{\begin{array}{l}
\frac{\mathrm{d}}{\mathrm{d} t} N(t)-d_{1} \Delta N(t)+f(\bar{N}(t))-f(\underline{N}(t)) \geq 0, \\
u(t) \geq \beta(\underline{N}(t)) \bar{N}(t)-\beta(\bar{N}(t)) \underline{N}(t) .
\end{array}\right.
$$

As the function $\beta$ is continuously differentiable and by using the mean value theorem, we obtain, for $t \in[0, \tau]$,

$$
f(\bar{N}(t))-f(\underline{N}(t))=f^{\prime}\left(\nu_{1}(t)\right) N(t),
$$

with $\nu_{1}(t)=\left(1-c_{1}(t)\right) \bar{N}(t)+c_{1}(t) \underline{N}(t)$, for some $c_{1}(t) \in[0,1]$ and

$$
\begin{aligned}
\beta(\underline{N}(t)) \bar{N}(t)-\beta(\bar{N}(t)) \underline{N}(t) & =\beta(\bar{N}(t))(\bar{N}(t)-\underline{N}(t))-\bar{N}(t)(\beta(\bar{N}(t)-\beta(\underline{N}(t)), \\
& =\beta(\bar{N}(t)) N(t)-\bar{N}(t) \beta^{\prime}\left(\nu_{2}(t)\right) N(t),
\end{aligned}
$$

with $\nu_{2}(t)=\left(1-c_{2}(t)\right) \bar{N}(t)+c_{2}(t) \underline{N}(t)$, for some $c_{2}(t) \in[0,1]$. Consequently, for $t \in[0, \tau]$,

$$
\left\{\begin{array}{l}
\frac{\mathrm{d}}{\mathrm{d} t} N(t)-d_{1} \Delta N(t)+f^{\prime}\left(\nu_{1}(t)\right) N(t) \geq 0 \\
u(t) \geq \beta(\bar{N}(t)) N(t)-\bar{N}(t) \beta^{\prime}\left(\nu_{2}(t)\right) N(t)
\end{array}\right.
$$

As in the proof of Proposition 2, we put $P(t)=e^{-\rho t} N(t)$. Then, for $\rho>0$ large enough, we obtain $P(t) \geq 0$ for $t \in[0, \tau]$. This leads to

$$
N(t)=\bar{N}(t)-\underline{N}(t) \geq 0, \quad \text { for } t \in[0, \tau] .
$$


Furthermore, as $\bar{N}(t) \geq \underline{N}(t) \geq 0$ for $t \in[0, \tau]$ and $\beta$ decreasing, we obtain

$$
u(t) \geq 0 \quad t \in[0, \tau] .
$$

This means that

$$
\underline{u}(t) \leq \bar{u}(t), \quad \text { for } t \in[0, \tau] .
$$

We repeat the same argument on the intervals $[\tau, 2 \tau],[2 \tau, 3 \tau], \ldots$, and we obtain

$$
\underline{N}(t) \leq \bar{N}(t) \quad \text { and } \quad \underline{u}(t) \leq \bar{u}(t), \quad \text { for } t \in[0,+\infty) .
$$

The proof is complete.

The next theorem gives the existence of solutions of (7). The positivity and uniqueness follow from the previous results.

Theorem 4.2. Let $\left(N_{0}, u_{0}\right) \in X^{+} \times C^{+}$. If (7) has nonnegative upper and lower solutions $(\bar{N}, \bar{u}),(\underline{N}, \underline{u})$ on $[0,+\infty)$, such that $0 \leq \underline{N}(0) \leq N_{0} \leq \bar{N}(0)$ and $0 \leq \underline{u}(\theta) \leq u_{0}(\theta) \leq \bar{u}(\theta)$, for all $-\tau \leq \theta \leq 0$, then it has a nonnegative solution $(N, u)$ defined on $[0,+\infty)$. Furthermore, $(N, u)$ satisfies

$$
0 \leq \underline{N}(t) \leq N(t) \leq \bar{N}(t) \quad \text { and } \quad 0 \leq \underline{u}(t) \leq u(t) \leq \bar{u}(t) \text {, for } t \in[0,+\infty) .
$$

Proof. We define two sequences $\left\{\left(\bar{N}^{k}, \bar{u}^{k}\right)\right\}_{k=1}^{+\infty}$ and $\left\{\left(\underline{N}^{k}, \underline{u}^{k}\right)\right\}_{k=1}^{+\infty}$ solutions on $[0,+\infty)$ of the following systems

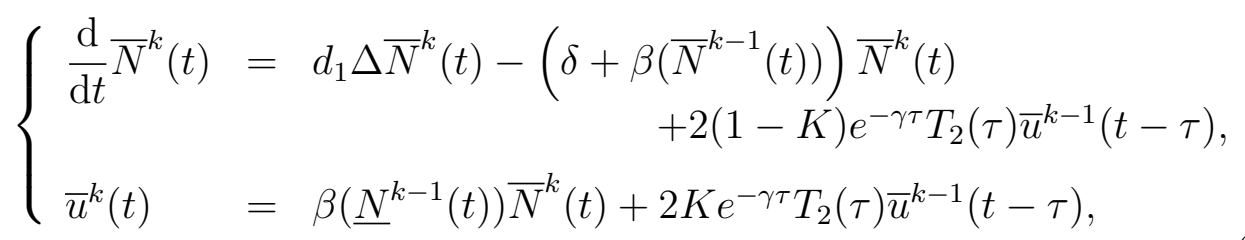

and

$$
\left\{\begin{aligned}
\frac{\mathrm{d}}{\mathrm{d} t} \underline{N}^{k}(t)= & d_{1} \Delta \underline{N}^{k}(t)-\left(\delta+\beta\left(\underline{N}^{k-1}(t)\right)\right) \underline{N}^{k}(t) \\
& +2(1-K) e^{-\gamma \tau} T_{2}(\tau) \underline{u}^{k-1}(t-\tau), \\
\underline{u}^{k}(t)= & \beta\left(\bar{N}^{k-1}(t)\right) \underline{N}^{k}(t)+2 K e^{-\gamma \tau} T_{2}(\tau) \underline{u}^{k-1}(t-\tau),
\end{aligned}\right.
$$

with, for $k=1,2, \ldots, \bar{N}^{k}(0)=\underline{N}^{k}(0)=N_{0}, \bar{u}^{k}(\theta)=\underline{u}^{k}(\theta)=u_{0}(\theta)$, $-\tau \leq \theta \leq 0, \bar{N}^{k}(t)(0)=\bar{N}^{k}(t)(\pi)=\bar{u}^{k}(t)(0)=\bar{u}^{k}(t)(\pi)=0, \underline{N}^{k}(t)(0)=$ 
$\underline{N}^{k}(t)(\pi)=\underline{u}^{k}(t)(0)=\underline{u}^{k}(t)(\pi)=0$, and for $k=0,\left(\underline{N}^{0}, \underline{u}^{0}\right)=(\underline{N}, \underline{u})$ and $\left(\bar{N}^{0}, \bar{u}^{0}\right)=(\bar{N}, \bar{u})$.

It is clear that the two sequences $\left\{\left(\bar{N}^{k}, \bar{u}^{k}\right)\right\}_{k=0}^{+\infty},\left\{\left(\underline{N}^{k}, \underline{u}^{k}\right)\right\}_{k=0}^{+\infty}$ are well defined. We will prove now by induction that $\left(\bar{N}^{k}, \bar{u}^{k}\right),\left(\underline{N}^{k}, \underline{u}^{k}\right)$ are upper and lower solutions of (7) and

$$
\left\{\begin{array}{l}
\underline{N}^{0} \leq \underline{N}^{1} \leq \ldots \leq \underline{N}^{k} \leq \bar{N}^{k} \leq \ldots \leq \bar{N}^{1} \leq \bar{N}^{0} \\
\underline{u}^{0} \leq \underline{u}^{1} \leq \ldots \leq \underline{u}^{k} \leq \bar{u}^{k} \leq \ldots \leq \bar{u}^{1} \leq \bar{u}^{0}
\end{array}\right.
$$

For $k=0$, we have $\left(\bar{N}^{0}, \bar{u}^{0}\right)=(\bar{N}, \bar{u})$ and $\left(\underline{N}^{0}, \underline{u}^{0}\right)=(\underline{N}, \underline{u})$. Then, $\left(\bar{N}^{0}, \bar{u}^{0}\right)$, $\left(\underline{N}^{0}, \underline{u}^{0}\right)$ are upper and lower solutions. Furthermore, by Proposition $3, \underline{N}^{0} \leq$ $\bar{N}^{0}$ and $\underline{u}^{0} \leq \bar{u}^{0}$. On the other hand, by (10) and (11), we have

$$
\left\{\begin{array}{l}
\frac{\mathrm{d}}{\mathrm{d} t} \bar{N}^{1}(t)=d_{1} \Delta \bar{N}^{1}(t)-\left(\delta+\beta\left(\bar{N}^{0}(t)\right)\right) \bar{N}^{1}(t)+2(1-K) e^{-\gamma \tau} T_{2}(\tau) \bar{u}^{0}(t-\tau), \\
\bar{u}^{1}(t)=\beta\left(\underline{N}^{0}(t)\right) \bar{N}^{1}(t)+2 K e^{-\gamma \tau} T_{2}(\tau) \bar{u}^{0}(t-\tau),
\end{array}\right.
$$

and

$$
\left\{\begin{array}{l}
\frac{\mathrm{d}}{\mathrm{d} t} \bar{N}^{0}(t) \geq d_{1} \Delta \bar{N}^{0}(t)-f\left(\bar{N}^{0}(t)\right)+2(1-K) e^{-\gamma \tau} T_{2}(\tau) \bar{u}^{0}(t-\tau), \\
\bar{u}^{0}(t) \geq \beta\left(\underline{N}^{0}(t)\right) \bar{N}^{0}(t)+2 K e^{-\gamma \tau} T_{2}(\tau) \bar{u}^{0}(t-\tau) .
\end{array}\right.
$$

Then, if we put $\hat{N}^{0}=\bar{N}^{1}-\bar{N}^{0}$ and $\hat{u}^{0}=\bar{u}^{1}-\bar{u}^{0}$, we obtain

$$
\left\{\begin{array}{l}
\frac{\mathrm{d}}{\mathrm{d} t} \hat{N}^{0}(t) \leq d_{1} \Delta \hat{N}^{0}(t)-\left(\delta+\beta\left(\bar{N}^{0}(t)\right)\right) \hat{N}^{0}(t) \\
\hat{u}^{0}(t) \\
\leq \beta\left(\underline{N}^{0}(t)\right) \hat{N}^{0}(t)
\end{array}\right.
$$

with $\hat{N}^{0}(0) \leq 0$ and $\hat{u}^{0}(\theta) \leq 0$, for $-\tau \leq \theta \leq 0$. Consequently, by the principle of maximum, we obtain $\hat{N}^{0} \leq 0$ and $\hat{u}^{0} \leq 0$. Thus, $\bar{N}^{0} \geq \bar{N}^{1}$ and $\bar{u}^{0} \geq \bar{u}^{1}$. Likewise, we prove that $\underline{N}^{0} \leq \underline{N}^{1}$ and $\underline{u}^{0} \leq \underline{u}^{1}$. Recall that the function $N \mapsto \beta(N)$ is decreasing. So, thanks to (13) and the fact that $\bar{N}^{0} \geq \bar{N}^{1}$ and $\bar{u}^{0} \geq \bar{u}^{1}$, we obtain

$$
\left\{\begin{array}{l}
\frac{\mathrm{d}}{\mathrm{d} t} \bar{N}^{1}(t) \geq d_{1} \Delta \bar{N}^{1}(t)-f\left(\bar{N}^{1}(t)\right)+2(1-K) e^{-\gamma \tau} T_{2}(\tau) \bar{u}^{1}(t-\tau), \\
\bar{u}^{1}(t) \geq \beta\left(\underline{N}^{1}(t)\right) \bar{N}^{1}(t)+2 K e^{-\gamma \tau} T_{2}(\tau) \bar{u}^{1}(t-\tau) .
\end{array}\right.
$$


Using the same technique as above, we obtain

$$
\left\{\begin{array}{l}
\frac{\mathrm{d}}{\mathrm{d} t} \underline{N}^{1}(t) \leq d_{1} \Delta \underline{N}^{1}(t)-f\left(\underline{N}^{1}(t)\right)+2(1-K) e^{-\gamma \tau} T_{2}(\tau) \underline{u}^{1}(t-\tau), \\
\underline{u}^{1}(t) \leq \beta\left(\bar{N}^{1}(t)\right) \underline{N}^{1}(t)+2 K e^{-\gamma \tau} T_{2}(\tau) \underline{u}^{1}(t-\tau) .
\end{array}\right.
$$

We proved that $\left(\bar{N}^{1}, \bar{u}^{1}\right),\left(\underline{N}^{1}, \underline{u}^{1}\right)$ are upper and lower solutions of $(7)$, with $\bar{N}^{0} \geq \bar{N}^{1}, \bar{u}^{0} \geq \bar{u}^{1}, \underline{N}^{0} \leq \underline{N}^{1}$ and $\underline{u}^{0} \leq \underline{u}^{1}$. Furthermore, Proposition 3 implies that $\underline{N}^{1} \leq \bar{N}^{1}$ and $\underline{u}^{1} \leq \bar{u}^{1}$. In the same way, we obtain by induction that, for all $k \in \mathbb{N}^{*},\left(\bar{N}^{k}, \bar{u}^{k}\right),\left(\underline{N}^{k}, \underline{u}^{k}\right)$ are upper and lower solutions with $\bar{N}^{k-1} \geq \bar{N}^{k}, \bar{u}^{k-1} \geq \bar{u}^{k}, \underline{N}^{k-1} \leq \underline{N}^{k}, \underline{u}^{k-1} \leq \underline{u}^{k}, \underline{N}^{k} \leq \bar{N}^{k}$ and $\underline{u}^{k} \leq \bar{u}^{k}$ (the two last inequalities are obtained by Proposition 3 ). We conclude that

$$
\left\{\begin{array}{l}
\underline{N}^{0} \leq \underline{N}^{1} \leq \ldots \leq \underline{N}^{k} \leq \bar{N}^{k} \leq \ldots \leq \bar{N}^{1} \leq \bar{N}^{0} \\
\underline{u}^{0} \leq \underline{u}^{1} \leq \ldots \leq \underline{u}^{k} \leq \bar{u}^{k} \leq \ldots \leq \bar{u}^{1} \leq \bar{u}^{0} .
\end{array}\right.
$$

Since the sequences $\left\{\left(\underline{N}^{k}, \underline{u}^{k}\right)\right\}_{k=0}^{+\infty}$ and $\left\{\left(\bar{N}^{k}, \bar{u}^{k}\right)\right\}_{k=0}^{+\infty}$ are monotone and bounded, their limits exist. We put, for $t \in[0,+\infty)$,

$\lim _{k \rightarrow+\infty}\left(\underline{N}^{k}(t), \underline{u}^{k}(t)\right)=(\underline{X}(t), \underline{v}(t)) \quad$ and $\quad \lim _{k \rightarrow+\infty}\left(\bar{N}^{k}(t), \bar{u}^{k}(t)\right)=(\bar{X}(t), \bar{v}(t))$.

It is clear that $\underline{X}(t) \leq \bar{X}(t)$ and $\underline{v}(t) \leq \bar{v}(t)$, for all $t \in[0,+\infty)$. On the other hand, we use the abstract integral formulations of systems (11)-(12) and we tend $k$ to $+\infty$. Then, we obtain

$$
\left\{\begin{array}{l}
\frac{\mathrm{d}}{\mathrm{d} t} \bar{X}(t)=d_{1} \Delta \bar{X}(t)-f(\bar{X}(t))+2(1-K) e^{-\gamma \tau} T_{2}(\tau) \bar{v}(t-\tau), \\
\bar{v}(t)=\beta(\underline{X}(t)) \bar{X}(t)+2 K e^{-\gamma \tau} T_{2}(\tau) \bar{v}(t-\tau),
\end{array}\right.
$$

and

$$
\left\{\begin{array}{l}
\frac{\mathrm{d}}{\mathrm{d} t} \underline{X}(t)=d_{1} \Delta \underline{X}(t)-f(\underline{X}(t))+2(1-K) e^{-\gamma \tau} T_{2}(\tau) \underline{v}(t-\tau), \\
\underline{v}(t)=\beta(\bar{X}(t)) \underline{X}(t)+2 K e^{-\gamma \tau} T_{2}(\tau) \underline{v}(t-\tau) .
\end{array}\right.
$$

Consequently, $(\underline{X}, \underline{v})$ (respectively, $(\bar{X}, \bar{v})$ ) is an upper (respectively, lower) solution of (7). Then, by Proposition 3, we have $\underline{X} \geq \bar{X}$ and $\underline{v} \geq \bar{v}$. Consequently, $\underline{X}=\bar{X}$ and $\underline{v}=\bar{v}$. We conclude that $(N, u)=(\underline{X}, \underline{v})=(\bar{X}, \bar{v})$ is a solution of (7). The proof is complete. 
The solution obtained by Theorem 4.2 is a classical solution in the sense that it is continuously differentiable with respect to $t$ and twice continuously differentiable with respect to $x$ (see the next proposition). This can be seen using the abstract integral representation (8) of the solutions and the following compatibility condition, satisfied by the initial condition $\left(N_{0}, \phi\right) \in X^{+} \times C^{+}$,

$$
\phi(0)=g\left(N_{0}\right)+2 K e^{-\gamma \tau} T_{2}(\tau) \phi(-\tau) .
$$

Under the condition (14), it is not difficult to see that $u$ is continuous. In fact, the piecewise function $N$ defined by (7), has a continuous first derivative for all $t>0$ and the function $u$ is continuous for all $t \geq-\tau$ if and only if the initial condition $\left(N_{0}, \phi\right) \in X^{+} \times C^{+}$satisfies (14). This is done by the following proposition.

Proposition 4. Let $(N, u)$ be the piecewise function defined by (7). Then, $N$ has a continuous first derivative for all $t>0$ and $u$ is continuous for all $t \geq-\tau$ if and only if the initial condition $\left(N_{0}, \phi\right) \in X^{+} \times C^{+}$satisfies the equality (14).

Proof. It is clear that the continuity at $t=0$ of $u$ says $\phi(0)=g\left(N_{0}\right)+$ $2 K e^{-\gamma \tau} T_{2}(\tau) \phi(-\tau)$. Conversely, assume that (14) is satisfied. Then,

$$
u(t)=\left\{\begin{array}{lr}
\beta(N(t)) N(t)+2 K e^{-\gamma \tau} T_{2}(\tau) \phi(t-\tau), & t \in(0, \tau], \\
\phi(t), & -\tau \leq t \leq 0,
\end{array}\right.
$$

is continuous at $t=0$. This implies the continuity of $u$ and the continuity of the derivative of $N$ at $t=0, \tau, 2 \tau, \ldots$.

Now, it suffice to prove the existence of a pair of upper and lower solutions to prove the existence of solutions of (7). Before starting to look for upper and lower solutions, we establish the following result.

Proposition 5. Assume that

$$
2 K e^{-\left(\gamma+d_{2}\right) \tau}>1
$$

Then, all nontrivial solutions of the system (7) with positive initial conditions are unbounded. 
Proof. Let us focus only on the component $u$. Let $t>0$. Consider the integer part $n=\left\lfloor\frac{t}{\tau}\right\rfloor$ of $\frac{t}{\tau}$. Then, $-\tau \leq t-(n+1) \tau<0$. Thanks to $N \neq 0$ and the expression of $u(t)(x):=u(t, x)$ in $(7)$, we get

$$
\begin{aligned}
\int_{0}^{\pi} v(x) u(t, x) d x & >2 K e^{-\gamma \tau} \int_{0}^{\pi}\left(\int_{0}^{\pi} v(x) \Gamma_{2}(\tau, x, y) d x\right) u(t-\tau, y) d y, \\
& =2 K e^{-\left(\gamma+d_{2}\right) \tau} \int_{0}^{\pi} v(x) u(t-\tau, x) d x \\
& >\left(2 K e^{-\left(\gamma+d_{2}\right) \tau}\right)^{2} \int_{0}^{\pi} v(x) u(t-2 \tau, x) d x \\
& >\left(2 K e^{-\left(\gamma+d_{2}\right) \tau}\right)^{n+1} \inf _{-\tau \leq \theta \leq 0}\left(\int_{0}^{\pi} v(x) u_{0}(\theta, x) d x\right),
\end{aligned}
$$

where $v(x)=\sin (x)$. Thanks to (15), we obtain

$$
\lim _{t \rightarrow+\infty} \int_{0}^{\pi} v(x) u(t, x) d x=+\infty .
$$

This implies that the solution is unbounded.

Throughout this paper, we assume

$$
2 K e^{-\left(\gamma+d_{2}\right) \tau}<1
$$

The condition (16) is necessary for the boundedness of solutions of (7) (see Proposition 5). Now, we will construct appropriate upper and lower solutions of $(7)$.

Theorem 4.3. We consider

$$
(\bar{N}(t)(x), \bar{u}(t)(x))=\left(\eta e^{\eta t} \sin (x), \frac{\beta(0)}{1-2 K e^{-\gamma \tau-d_{2} \tau-\eta \tau}} \eta e^{\eta t} \sin (x)\right), \quad x \in[0, \pi] .
$$

Then, for $\eta>0$ large enough, $(\bar{N}, \bar{u})$ (respectively, $(\underline{N}, \underline{u})=(0,0)$ ) is an upper (respectively, lower) solution of $(7)$ on $[0,+\infty)$.

Proof. First, we can always choose $(\underline{N}, \underline{u})=(0,0)$ as a lower solution. Second, using the fact that

$$
\int_{0}^{\pi} \Gamma_{2}(\tau, x, s) \sin (s) d s=e^{-d_{2} \tau} \sin (x)
$$


we have

$$
\begin{aligned}
\frac{\mathrm{d}}{\mathrm{d} t} \bar{N}(t) & -d_{1} \Delta \bar{N}(t)+f(\bar{N}(t))-2(1-K) e^{-\gamma \tau} T_{2}(\tau) \bar{u}(t-\tau) \\
& \geq\left[\eta+d_{1}+\delta-2(1-K) e^{-\gamma \tau} e^{-d_{2} \tau} \frac{\beta(0) e^{-\eta \tau}}{1-2 K e^{-\gamma \tau-d_{2} \tau-\eta \tau}}\right] \eta e^{\eta t} \sin (x)
\end{aligned}
$$

and

$$
\begin{aligned}
\bar{u}(t) & -\beta(\underline{N}) \bar{N}(t)-2 K e^{-\gamma \tau} T_{2}(\tau) \bar{u}(t-\tau) \\
& =\left[\frac{\beta(0)}{1-2 K e^{-\gamma \tau-d_{2} \tau-\eta \tau}}-\beta(0)-\frac{2 K e^{-\gamma \tau} e^{-d_{2} \tau} \beta(0) e^{-\eta \tau}}{1-2 K e^{-\gamma \tau-d_{2} \tau-\eta \tau}}\right] \eta e^{\eta t} \sin (x), \\
& =0 .
\end{aligned}
$$

If we choose $\eta>0$ such that

$$
\eta \geq-d_{1}-\delta+2(1-K) e^{-\gamma \tau} e^{-d_{2} \tau} \frac{\beta(0)}{1-2 K e^{-\gamma \tau-d_{2} \tau}},
$$

we obtain a suitable upper solution $(\bar{N}, \bar{u})$. The proof is completed.

The following theorem states the existence result for the problem (7), which is a direct consequence of Theorems 4.2 and 4.3 .

Theorem 4.4. Consider $\bar{N}$ and $\bar{u}$ the functions defined in Theorem 4.3. Let $\left(N_{0}, u_{0}\right) \in X^{+} \times C^{+}$be such that $0 \leq N_{0}(x) \leq \bar{N}(0)(x)$, for $x \in[0, \pi]$, and $0 \leq u_{0}(\theta, x) \leq \bar{u}(\theta)(x)$, for $-\tau \leq \theta \leq 0$ and $x \in[0, \pi]$ ( $\eta>0$ is chosen as in Theorem 4.3), with $N_{0}(0)=N_{0}(\pi)=0$ and $u(\theta, 0)=u(\theta, \pi)=0$, for $-\tau \leq \theta \leq 0$. Then, there exists a unique nonnegative solution $(N, u)$ of $(7)$. Furthermore, $(N, u)$ satisfies

$$
0 \leq N(t) \leq \bar{N}(t), \quad 0 \leq u(t) \leq \bar{u}(t), \text { for all } t \geq 0 .
$$

\section{Global attractivity of the trivial steady state}

Let $v(x):=\sin (x)$, for $x \in[0, \pi]$. Consider the following eigenvalue problem associated to $v(x)$

$$
\left\{\begin{array}{l}
\lambda_{0} v(x)=d_{1} \frac{d^{2} v(x)}{d x^{2}}-(\delta+\beta(0)) v(x)+\frac{2(1-K) e^{-\gamma \tau-d_{2} \tau}}{1-2 K e^{-\gamma \tau-d_{2} \tau}} \beta(0) v(x), \\
v(0)=v(\pi)=0
\end{array}\right.
$$


Then, we have

$$
\lambda_{0}=-d_{1}-\delta+\left(\frac{2 e^{-\gamma \tau-d_{2} \tau}-1}{1-2 K e^{-\gamma \tau-d_{2} \tau}}\right) \beta(0)
$$

Now, we are in position to prove the following theorem.

Theorem 5.1. Assume that $\lambda_{0}<0$, where $\lambda_{0}$ is given by (18). Then, the zero steady state of $(7)$ is globally attractive.

Proof. Let consider the Lyapunov functional on $X^{+} \times C^{+}$

$$
V(w, \phi):=\int_{0}^{\pi} v(x) w(x) d x+\frac{2(1-K) e^{-\gamma \tau-d_{2} \tau}}{1-2 K e^{-\gamma \tau-d_{2} \tau}} \int_{-\tau}^{0} \int_{0}^{\pi} v(x) \phi(\theta)(x) d x d \theta .
$$

We define the following distance function on $X$

$$
d(y, z):=\int_{0}^{\pi} v(x)|y(x)-z(x)| d x .
$$

Then, for $(w, \phi) \in X^{+} \times C^{+}$, we have

$$
V(w, \phi) \geq \int_{0}^{\pi} v(x) w(x) d x=d(w, 0) .
$$

The derivative of $V$ along the solution trajectory $t \mapsto\left(N(t), u_{t}\right)$ of $(7)$ is calculated as follows (remember that we use the notations $N(t)(x):=N(t, x)$, $u(t)(x):=u(t, x)$ and $\left.u_{t}(\theta)(x)=u_{t}(\theta, x)=u(t+\theta, x)\right)$ :

$$
\begin{aligned}
\dot{V}\left(N(t), u_{t}\right)= & \int_{0}^{\pi} v(x)\left[d_{1} \frac{\partial^{2} N(t, x)}{\partial x^{2}}-(\delta+\beta(N(t, x))) N(t, x)\right] d x \\
& +2(1-K) e^{-\gamma \tau} \int_{0}^{\pi} v(x) \int_{0}^{\pi} \Gamma_{2}(\tau, x, y) u(t-\tau, y) d y d x \\
& +\frac{2(1-K) e^{-\gamma \tau-d_{2} \tau}}{1-2 K e^{-\gamma \tau-d_{2} \tau}} \int_{0}^{\pi} v(x)[\beta(N(t, x)) N(t, x) \\
& \left.+2 K e^{-\gamma \tau} \int_{0}^{\pi} \Gamma_{2}(\tau, x, y) u(t-\tau, y) d y-u(t-\tau, x)\right] d x \\
= & \int_{0}^{\pi}\left[d_{1} \frac{d^{2} v(x)}{d x^{2}}-(\delta+\beta(N(t, x))) v(x)\right. \\
& \left.+\frac{2(1-K) e^{-\gamma \tau-d_{2} \tau}}{1-2 K e^{-\gamma \tau-d_{2} \tau}} \beta(N(t, x)) v(x)\right] N(t, x) d x .
\end{aligned}
$$


Hence, using (17) we obtain

$\dot{V}\left(N(t), u_{t}\right)=\int_{0}^{\pi} v(x)\left[\lambda_{0}+\frac{1-2 e^{-\gamma \tau-d_{2} \tau}}{1-2 K e^{-\gamma \tau-d_{2} \tau}}(\beta(0)-\beta(N(t, x)))\right] N(t, x) d x$.

Note that $\beta(0)-\beta(N(t, x)) \geq 0$ since $\beta$ is a decreasing function. Remember that the condition (16) is satisfied. We consider two cases.

- If $1-2 e^{-\gamma \tau-d_{2} \tau} \leq 0$, then we have

$$
\dot{V}\left(N(t), u_{t}\right) \leq \lambda_{0} \int_{0}^{\pi} v(x) N(t, x) d x \leq 0
$$

- If $1-2 e^{-\gamma \tau-d_{2} \tau}>0$, then we have

$$
\begin{aligned}
\dot{V}\left(N(t), u_{t}\right) & \leq \int_{0}^{\pi} v(x)\left[\lambda_{0}+\frac{1-2 e^{-\gamma \tau-d_{2} \tau}}{1-2 K e^{-\gamma \tau-d_{2} \tau}} \beta(0)\right] N(t, x) d x, \\
& =-\left(d_{1}+\delta\right) \int_{0}^{\pi} v(x) N(t, x) d x \leq 0 .
\end{aligned}
$$

Consequently, we get

$$
\dot{V}\left(N(t), u_{t}\right) \leq-\underline{\delta} d(N(t, \cdot), 0) \leq 0,
$$

where $\underline{\delta}:=\min \left\{-\lambda_{0}, d_{1}+\delta\right\}>0$.

Then, the function $t \mapsto V\left(N(t), u_{t}\right)$ is nonincreasing and we have

$$
V\left(N(t), u_{t}\right) \underset{t \rightarrow+\infty}{\longrightarrow} \inf _{s \geq 0} V\left(N(s), u_{s}\right)=: \alpha \in \mathbb{R}^{+} .
$$

Furthermore, by integrating (20) we get

$$
\underline{\delta} \int_{0}^{t} d(N(s, \cdot), 0) d s \leq V\left(N(0), u_{0}\right)-V\left(N(t), u_{t}\right) .
$$

The both sides of the inequality (21) are nondecreasing functions. Then, the limits exist and satisfy

$$
\lim _{t \rightarrow+\infty} \int_{0}^{t} d(N(s, \cdot), 0) d s \leq \frac{1}{\underline{\delta}}\left[V\left(N(0), u_{0}\right)-\alpha\right] .
$$


Since $\dot{V}\left(N(t), u_{t}\right) \leq 0$, we have $V\left(N(t), u_{t}\right) \leq V\left(N(0), u_{0}\right)$ for all $t \geq 0$. This implies that $d(N(t, \cdot), 0) \leq d(N(0, \cdot), 0)$ for all $t \geq 0$. Moreover, for all $t \geq 0$,

$$
\begin{aligned}
d(u(t, \cdot), 0) & =\int_{0}^{\pi} v(x)\left[\beta(N(t, x)) N(t, x)+2 K e^{-\gamma \tau} \int_{0}^{\pi} \Gamma_{2}(\tau, x, y) u(t-\tau, y) d y\right] d x \\
& \leq \beta(0) d(N(t, \cdot), 0)+2 K e^{-\gamma \tau-d_{2} \tau} \int_{0}^{\pi} v(x) u(t-\tau, x) d x \\
& \leq \beta(0) d(N(0, \cdot), 0)+2 K e^{-\gamma \tau-d_{2} \tau} d(u(t-\tau, \cdot), 0) .
\end{aligned}
$$

Since $2 K e^{-\gamma \tau-d_{2} \tau}<1$, this last inequality implies that

$$
d(u(t, \cdot), 0) \leq \beta(0) d(N(0, \cdot), 0) \sum_{n=0}^{+\infty}\left(2 K e^{-\gamma \tau-d_{2} \tau}\right)^{n}=\frac{\beta(0) d(N(0, \cdot), 0)}{1-2 K e^{-\gamma \tau-d_{2} \tau}} .
$$

Thus, we have

$$
\begin{aligned}
\left|\frac{d}{d t} d(N(t, \cdot), 0)\right|= & \mid \int_{0}^{\pi}\left[d_{1} \frac{d^{2} v(x)}{d x^{2}}-(\delta+\beta(N(t, x))) v(x)\right] N(t, x) d x \\
& +2(1-K) e^{-\gamma \tau-d_{2} \tau} \int_{0}^{\pi} v(x) u(t-\tau, x) d x \mid, \\
\leq & \mid \int_{0}^{\pi}\left[\lambda_{0} v(x)+(\beta(0)-\beta(N(t, x))) v(x)-\frac{2(1-K) e^{-\gamma \tau-d_{2} \tau}}{1-2 K e^{-\gamma \tau-d_{2} \tau}} \beta(0) v(x)\right] \\
& \quad \times N(t, x) d x \mid+2(1-K) e^{-\gamma \tau-d_{2} \tau} d(u(t-\tau, \cdot), 0), \\
\leq & \max \left(\beta(0),-\lambda_{0}+\frac{2(1-K) e^{-\gamma \tau-d_{2} \tau}}{1-2 K e^{-\gamma \tau-d_{2} \tau}} \beta(0)\right) d(N(t, \cdot), 0) \\
& +2(1-K) e^{-\gamma \tau-d_{2} \tau} d(u(t-\tau, \cdot), 0), \\
\leq & \max \left(\beta(0),-\lambda_{0}+\frac{2(1-K) e^{-\gamma \tau-d_{2} \tau}}{1-2 K e^{-\gamma \tau-d_{2} \tau}} \beta(0)\right) d(N(0, \cdot), 0) \\
& +2(1-K) e^{-\gamma \tau-d_{2} \tau} \frac{\beta(0) d(N(0, \cdot), 0)}{1-2 K e^{-\gamma \tau-d_{2} \tau}} .
\end{aligned}
$$

This implies that $(d / d t) d(N(t, \cdot), 0)$ is uniformly bounded. Then, $d(N(t, \cdot), 0)$ is uniformly continuous. The Barbalat's Lemma applied to the function $t \mapsto \int_{0}^{t} d(N(s, \cdot), 0) d s$ shows that

$$
\lim _{t \rightarrow+\infty} d(N(t, \cdot), 0)=0
$$


Furthermore, we have

$$
\begin{aligned}
& d(u(t), 0)=\int_{0}^{\pi} v(x) u(t, x) d x \\
& =\int_{0}^{\pi} v(x)\left[\beta(N(t, x)) N(t, x)+2 K e^{-\gamma \tau} \int_{0}^{\pi} \Gamma_{2}(\tau, x, y) u(t-\tau, y) d y\right] d x \\
& \leq \beta(0) d(N(t), 0)+2 K e^{-\gamma \tau-d_{2} \tau} d(u(t-\tau), 0)
\end{aligned}
$$

Hence, for $n=\left\lfloor\frac{t}{\tau}\right\rfloor \in \mathbb{N}$, we have

$$
\begin{aligned}
d(u(t), 0) \leq & \beta(0) \sum_{m=0}^{n}\left(2 K e^{-\gamma \tau-d_{2} \tau}\right)^{m} d(N(t-m \tau), 0) \\
& +\beta(0)\left(2 K e^{-\gamma \tau-d_{2} \tau}\right)^{n+1} d\left(u_{0}(t-(n+1) \tau), 0\right)
\end{aligned}
$$

From (16) and (22), the right-hand side of the above inequality tends to zero as $t \rightarrow+\infty$. That is

$$
d(u(t), 0) \rightarrow 0 \text { as } t \rightarrow+\infty
$$

Then, (22) and (23) imply the global attractivity of the zero steady state. This completes the proof.

Now, we prove a result dealing with the instability of the trivial steady state.

Theorem 5.2. Assume that $\lambda_{0}>0$, where $\lambda_{0}$ is given by (18). Then, no nontrivial solution of the system (7) tends to zero.

Proof. Let $(N, u)$ be a solution of (7). Assume by contradiction that $N(t, x) \rightarrow$ 0 as $t \rightarrow+\infty$, for $x \in[0, \pi]$. By the continuity of the function $\beta$, we have $\beta(N(t, x)) \rightarrow \beta(0)$ as $t \rightarrow+\infty$. Consequently, for all $\epsilon>0$ there exists $\sigma(\epsilon)$ such that, for all $t \geq \sigma(\epsilon)$ and $x \in[0, \pi]$,

$$
\beta(0)-\epsilon<\beta(N(t, x)) \leq \beta(0)
$$


We fix $0<\epsilon<\beta(0)$. We integrate the system (7) over $t \in(\sigma(\epsilon),+\infty)$,

$$
\begin{aligned}
\int_{0}^{\pi} v(x) N( & \sigma(\epsilon), x) d x=-d_{1} \int_{0}^{\pi} v(x) \int_{\sigma(\epsilon)}^{+\infty} \frac{\partial^{2} N}{\partial x^{2}}(t, x) d t d x \\
& +\int_{0}^{\pi} v(x) \int_{\sigma(\epsilon)}^{+\infty}[\delta+\beta(N(t, x))] N(t, x) d t d x \\
& -2(1-K) e^{-\gamma \tau} \int_{0}^{\pi} v(x) \int_{0}^{\pi} \Gamma_{2}(\tau, x, y) \int_{\sigma(\epsilon)}^{+\infty} u(t-\tau, y) d t d y d x
\end{aligned}
$$

and

$$
\begin{array}{r}
\int_{0}^{\pi} v(x) \int_{\sigma(\epsilon)}^{+\infty} u(t, x) d t d x=\int_{0}^{\pi} v(x) \int_{\sigma(\epsilon)}^{+\infty} \beta(N(t, x)) N(t, x) d t d x \\
+2 K e^{-\gamma \tau} \int_{0}^{\pi} v(x) \int_{0}^{\pi} \Gamma_{2}(\tau, x, y) \int_{\sigma(\epsilon)}^{+\infty} u(t-\tau, y) d t d y d x
\end{array}
$$

By applying Fubini's theorem, the last expression gives

$$
\begin{aligned}
\int_{0}^{\pi} v(x) \int_{0}^{\pi} \Gamma_{2}(\tau, x, y) \int_{\sigma(\epsilon)}^{+\infty} u(t-\tau, y) d t d y d x \\
=\int_{0}^{\pi} v(x) \int_{0}^{\pi} \Gamma_{2}(\tau, x, y) \int_{\sigma(\epsilon)-\tau}^{+\infty} u(t, y) d t d y d x \\
\geq \int_{0}^{\pi} v(x) \int_{0}^{\pi} \Gamma_{2}(\tau, x, y) \int_{\sigma(\epsilon)}^{+\infty} u(t, y) d t d y d x \\
\geq e^{-d_{2} \tau} \int_{0}^{\pi} v(x) \int_{\sigma(\epsilon)}^{+\infty} u(t, x) d t d x
\end{aligned}
$$

From (24), (26) and the inequality (27), it follows

$$
\begin{aligned}
\int_{0}^{\pi} v(x) \int_{\sigma(\epsilon)}^{+\infty} u(t, x) d t d x & \geq(\beta(0)-\epsilon) \int_{0}^{\pi} v(x) \int_{\sigma(\epsilon)}^{+\infty} N(t, x) d t d x \\
& +2 K e^{-\gamma \tau-d_{2} \tau} \int_{0}^{\pi} v(x) \int_{\sigma(\epsilon)}^{+\infty} u(t, x) d t d x .
\end{aligned}
$$

Then, we obtain

$$
\int_{0}^{\pi} v(x) \int_{\sigma(\epsilon)}^{+\infty} u(t, x) d t d x \geq \frac{\beta(0)-\epsilon}{1-2 K e^{-\gamma \tau-d_{2} \tau}} \int_{0}^{\pi} v(x) \int_{\sigma(\epsilon)}^{+\infty} N(t, x) d t d x .
$$


We deduce from (25) and (27), that

$$
\begin{aligned}
\int_{0}^{\pi} v(x) N(\sigma(\epsilon), x) d x \leq & d_{1} \int_{0}^{\pi} v(x) \int_{\sigma(\epsilon)}^{+\infty} N(t, x) d t d x \\
& +(\delta+\beta(0)) \int_{0}^{\pi} v(x) \int_{\sigma(\epsilon)}^{+\infty} N(t, x) d t d x \\
& -2(1-K) e^{-\gamma \tau-d_{2} \tau} \int_{0}^{\pi} v(x) \int_{\sigma(\epsilon)}^{+\infty} u(t, x) d t d x \\
\leq & \left(d_{1}+\delta+\beta(0)\right) \int_{0}^{\pi} v(x) \int_{\sigma(\epsilon)}^{+\infty} N(t, x) d t d x \\
& -\frac{2(1-K) e^{-\gamma \tau-d_{2} \tau}(\beta(0)-\epsilon)}{1-2 K e^{-\gamma \tau-d_{2} \tau}} \int_{0}^{\pi} v(x) \int_{\sigma(\epsilon)}^{+\infty} N(t, x) d t d x \\
= & -\left(\lambda_{0}-\frac{2(1-K) e^{-\gamma \tau-d_{2} \tau}}{1-2 K e^{-\gamma \tau-d_{2} \tau}}\right) \int_{0}^{\pi} v(x) \int_{\sigma(\epsilon)}^{+\infty} N(t, x) d t d x .
\end{aligned}
$$

Since $\lambda_{0}>0$, one can choose $\epsilon \in(0, \beta(0))$ small enough such that

$$
\int_{0}^{\pi} v(x) N(\sigma(\epsilon), x) d x<0 .
$$

This leads to a contradiction with the nonnegativity of the solutions and the fact that $v(x):=\sin (x)>0$, for $x \in(0, \pi)$. Then, $N(t, x)$ does not tend to zero as $t \rightarrow+\infty$.

\section{Existence and uniqueness of positive steady state}

To investigate the existence of positive steady state of (7), we consider, for $x \in[0, \pi]$, the following stationary problem

$$
\left\{\begin{array}{l}
0=d_{1} \frac{d^{2} N(x)}{d x^{2}}-(\delta+\beta(N(x))) N(x)+2(1-K) e^{-\gamma \tau} \int_{0}^{\pi} \Gamma_{2}(\tau, x, y) u(y) d y \\
u(x)=\beta(N(x)) N(x)+2 K e^{-\gamma \tau} \int_{0}^{\pi} \Gamma_{2}(\tau, x, y) u(y) d y \\
N(0)=N(\pi)=u(0)=u(\pi)=0 .
\end{array}\right.
$$


Suppose that $K \neq 0$ and define the following linear operator $\Phi: X \rightarrow X$, by

$$
\Phi(\varphi)(x):=2 K e^{-\gamma \tau} \int_{0}^{\pi} \Gamma_{2}(\tau, x, y) \varphi(y) d y, \quad \varphi \in X .
$$

By the Krein-Rutman theorem (see Theorem 3.2 of [31]), we can easily see that the spectral radius $r(\Phi)$ of the operator $\Phi$ is a simple positive eigenvalue with a positive eigenvector and there is no other eigenvalue with a positive eigenvector. As in the previous sections, we consider the function $v(x):=$ $\sin (x)$, for $x \in[0, \pi]$. Then, we have

$$
\Phi(v)(x)=2 K e^{-\gamma \tau} \int_{0}^{\pi} \Gamma_{2}(t, x, y) v(y) d y=2 K e^{-\gamma \tau-d_{2} \tau} v(x) .
$$

We conclude that

$$
r(\Phi)=2 K e^{-\left(\gamma+d_{2}\right) \tau}<1 .
$$

As a consequent, the operator $I d-\Phi$ is invertible and its inverse is given by

$$
\left((I d-\Phi)^{-1} \varphi\right)(x)=\varphi(x)+\sum_{n=1}^{+\infty}\left(\Phi^{n} \varphi\right)(x), \quad x \in[0, \pi], \varphi \in X .
$$

Then, from the second equation of $(28), u(x)$ can be written as follows

$$
u(x)=\beta(N(x)) N(x)+\sum_{n=1}^{+\infty} \Phi^{n}(\beta(N(\cdot)) N(\cdot))(x), \quad x \in[0, \pi] .
$$

Furthermore, thanks to (30), we have $u(0)=u(\pi)=0$ whenever $N(0)=$ $N(\pi)=0$. Then, the system (28) is equivalent (for $0<K \leq 1$ ) to

$$
\left\{\begin{array}{l}
0=d_{1} \frac{d^{2} N(x)}{d x^{2}}-(\delta+\beta(N(x))) N(x)+\frac{1-K}{K} \sum_{n=1}^{+\infty} \Phi^{n}(\beta(N(\cdot)) N(\cdot))(x), \\
u(x)=\beta(N(x)) N(x)+\sum_{n=1}^{+\infty} \Phi^{n}(\beta(N(\cdot)) N(\cdot))(x), \\
N(0)=N(\pi)=0 .
\end{array}\right.
$$

To get an explicit expression of $(I d-\Phi)^{-1}$, we consider the operator

$$
\left(T_{2}(t) \varphi\right)(x):=\int_{0}^{\pi} \Gamma_{2}(t, x, y) \varphi(y) d y, \quad \varphi \in X .
$$


In fact, $\left(T_{2}(t)\right)_{t \geq 0}$ is the linear semigroup on $X$ generated by $\partial_{t} v=d_{2} \Delta v$ with Dirichlet boundary condition. Then, for $\varphi \in X, n \in \mathbb{N}$ and $x \in[0, \pi]$, we have

$$
\left(\Phi^{n} \varphi\right)(x)=\left(2 K e^{-\gamma \tau}\right)^{n}\left(T_{2}(n \tau) \varphi\right)(x) .
$$

Consequently,

$$
\begin{aligned}
\left((I d-\Phi)^{-1} \varphi\right)(x) & =\varphi(x)+\sum_{n=1}^{+\infty}\left(\Phi^{n} \varphi\right)(x) \\
& =\varphi(x)+\int_{0}^{\pi} \sum_{n=1}^{+\infty}\left(2 K e^{-\gamma \tau}\right)^{n} \Gamma_{2}(n \tau, x, y) \varphi(y) \mathrm{d} y \\
& =\varphi(x)+\int_{0}^{\pi} \kappa(\tau, x, y) \varphi(y) \mathrm{d} y
\end{aligned}
$$

where

$$
\kappa(\tau, x, y)=\sum_{n=1}^{+\infty} \kappa_{n}(\tau, x, y), \quad x, y \in[0, \pi]
$$

and

$$
\kappa_{n}(\tau, x, y)=\left(2 K e^{-\gamma \tau}\right)^{n} \Gamma_{2}(n \tau, x, y), \quad x, y \in[0, \pi], n \in \mathbb{N}^{*} .
$$

Then, from the second equation of (28), we can write $u(x)$ as follows

$$
u(x)=\beta(N(x)) N(x)+\int_{0}^{\pi} \kappa(\tau, x, y) \beta(N(y)) N(y) d y .
$$

Thus, the system (28) is equivalent, for $0<K \leq 1$, to

$$
\left\{\begin{aligned}
& 0=d_{1} \frac{d^{2} N(x)}{d x^{2}}-(\delta+\beta(N(x))) N(x) \\
&+\frac{1-K}{K} \int_{0}^{\pi} \kappa(\tau, x, y) \beta(N(y)) N(y) d y \\
& u(x)=\beta(N(x)) N(x)+\int_{0}^{\pi} \kappa(\tau, x, y) \beta(N(y)) N(y) d y \\
& N(0)=N(\pi)=0 .
\end{aligned}\right.
$$

We need the following lemma.

Lemma 6.1. For any $M>0$, the series $\sum_{n=1}^{+\infty} \Phi^{n}(\beta(M v(\cdot)) M v(\cdot))(x)$ converges for all $x \in[0, \pi]$. 
Proof. We have, for $x \in[0, \pi]$,

$$
\sum_{n=1}^{+\infty} \Phi^{n}(\beta(M v(\cdot)) M v(\cdot))(x) \leq \beta(0) M \sum_{n=1}^{+\infty} \Phi^{n}(v)(x),
$$

Recalling that

$$
\Phi^{n}(v)=\left(2 K e^{-\gamma \tau-d_{2} \tau}\right)^{n} v, \quad n \in \mathbb{N}^{*} .
$$

Hence, from (16) and (32), we have

$$
\begin{aligned}
\sum_{n=1}^{+\infty} \Phi^{n}(\beta(M v(\cdot)) M v(\cdot))(x) & \leq \beta(0) M \sum_{n=1}^{+\infty}\left(2 K e^{-\gamma \tau-d_{2} \tau}\right)^{n} v(x) \\
& <\frac{\beta(0)}{1-2 K e^{-\gamma \tau-d_{2} \tau}} M v(x)<+\infty
\end{aligned}
$$

This completes the proof.

Using (30), the stationary problem (28) can be written as follows

$$
\left\{\begin{array}{l}
0=d_{1} \frac{d^{2} N(x)}{d x^{2}}-(\delta+\beta(N(x))) N(x)+\frac{1-K}{K} \sum_{n=1}^{+\infty} \Phi^{n}(\beta(N(\cdot)) N(\cdot))(x), \\
N(0)=N(\pi)=0 .
\end{array}\right.
$$

In order to prove the existence of positive stationary solution $N(x)$ of $(33)$, we first construct an upper and a lower solution $\bar{N}(x), \underline{N}(x)$ of $(33)$ :

$$
\left\{\begin{array}{l}
0 \geq d_{1} \frac{d^{2} \bar{N}(x)}{d x^{2}}-(\delta+\beta(\bar{N}(x))) \bar{N}(x)+\frac{1-K}{K} \sum_{n=1}^{+\infty} \Phi^{n}(\beta(\bar{N}(\cdot)) \bar{N}(\cdot))(x), \\
0 \leq d_{1} \frac{d^{2} \underline{N}(x)}{d x^{2}}-(\delta+\beta(\underline{N}(x))) \underline{N}(x)+\frac{1-K}{K} \sum_{n=1}^{+\infty} \Phi^{n}(\beta(\underline{N}(\cdot)) \underline{N}(\cdot))(x), \\
\bar{N}(0)=\bar{N}(\pi)=\underline{N}(0)=\underline{N}(\pi)=0 .
\end{array}\right.
$$

One can remark that, the definition of upper and lower solutions for the stationary problem (33) is different from the one given by the definition 4.1. On the existence of lower solution, we prove the following proposition.

Proposition 6. Assume that $\lambda_{0}>0$. Then, there exists a sufficiently small $\epsilon>0$ such that $\underline{N}(x)=\epsilon v(x)$ is a lower solution of (33). 
Proof. Let $\underline{N}(x):=\epsilon v(x)=\epsilon \sin (x)$. It is clear that $\underline{N}(0)=\underline{N}(\pi)=0$. We have

$$
\begin{aligned}
& d_{1} \frac{d^{2} \underline{N}(x)}{d x^{2}}-(\delta+\beta(\underline{N}(x))) \underline{N}(x)+\frac{1-K}{K} \sum_{n=1}^{+\infty} \Phi^{n}(\beta(\underline{N}(\cdot)) \underline{N}(\cdot))(x) \\
& =-d_{1} \epsilon v(x)-(\delta+\beta(\epsilon v(x))) \epsilon v(x)+\frac{1-K}{K} \sum_{n=1}^{+\infty} \Phi^{n}(\beta(\epsilon v(\cdot)) \epsilon v(\cdot))(x), \\
& \geq-d_{1} \epsilon v(x)-(\delta+\beta(0)) \epsilon v(x)+\frac{1-K}{K} \beta(\epsilon) \epsilon \sum_{n=1}^{+\infty} \Phi^{n}(v)(x), \\
& =-d_{1} \epsilon v(x)-(\delta+\beta(0)) \epsilon v(x)+\frac{1-K}{K} \beta(\epsilon) \epsilon \sum_{n=1}^{+\infty}\left(2 K e^{-\gamma \tau-d_{2} \tau}\right)^{n} v(x), \\
& =\left[-d_{1}-\delta-\beta(0)+\frac{2(1-K) e^{-\gamma \tau-d_{2} \tau}}{1-2 K e^{-\gamma \tau-d_{2} \tau}} \beta(\epsilon)\right] \epsilon v(x) .
\end{aligned}
$$

Note that

$$
-d_{1}-\delta-\beta(0)=\lambda_{0}-\frac{2(1-K) e^{-\gamma \tau-d_{2} \tau}}{1-2 K e^{-\gamma \tau-d_{2} \tau}} \beta(0) .
$$

Hence, from (34), we have

$$
\begin{aligned}
& d_{1} \frac{d^{2} \underline{N}(x)}{d x^{2}}-(\delta+\beta(\underline{N}(x))) \underline{N}(x)+\frac{1-K}{K} \sum_{n=1}^{+\infty} \Phi^{n}(\beta(\underline{N}(\cdot)) \underline{N}(\cdot))(x) \\
& \geq\left[\lambda_{0}-\frac{2(1-K) e^{-\gamma \tau-d_{2} \tau}}{1-2 K e^{-\gamma \tau-d_{2} \tau}}(\beta(0)-\beta(\epsilon))\right] \epsilon v(x), \\
& \geq 0
\end{aligned}
$$

for sufficiently small $\epsilon>0$ (by virtue of the continuity of $\beta$ ). Hence, $\underline{N}(x)=$ $\epsilon v(x)$ is a lower solution of (33).

To prove the existence of an upper solution, we need the following additional condition,

$$
\beta^{\star}:=\sup _{s \geq 0}[\beta(s) s]<+\infty .
$$

Under the condition (35), we have the following lemma. 
Lemma 6.2. Assume that (35) holds. Then, for any $M>0$, we have

$$
\sum_{n=1}^{+\infty} \Phi^{n}(\beta(M v(\cdot)) M v(\cdot))(x) \leq \frac{8 \beta^{\star} K e^{-\gamma \tau}}{\pi\left(1-2 K e^{-\gamma \tau-d_{2} \tau}\right)}\left(\sum_{k=1}^{+\infty} e^{-(2 k-1)^{2} d_{2} \tau}\right) v(x) .
$$

Proof. From (35), we have

$$
\begin{aligned}
\Phi(\beta(M v(\cdot)) M v(\cdot))(x) \leq & \beta^{\star} 2 K e^{-\gamma \tau} \int_{0}^{\pi} \Gamma_{2}(\tau, x, y) d y \\
\leq & \frac{8 \beta^{\star} K e^{-\gamma \tau}}{\pi} \sum_{k=1}^{+\infty} e^{-(2 k-1)^{2} d_{2} \tau} v(x) \\
\Phi^{2}(\beta(M v(\cdot)) M v(\cdot))(x) \leq & \frac{8 \beta^{\star} K e^{-\gamma \tau}}{\pi} \sum_{k=1}^{+\infty} e^{-(2 k-1)^{2} d_{2} \tau} 2 K e^{-\gamma \tau} \int_{0}^{\pi} \Gamma_{2}(\tau, x, y) v(y) d y, \\
= & \frac{8 \beta^{\star} K e^{-\gamma \tau}}{\pi} \sum_{k=1}^{+\infty} e^{-(2 k-1)^{2} d_{2} \tau} 2 K e^{-\gamma \tau-d_{2} \tau} v(x), \\
& \vdots \\
\Phi^{n}(\beta(M v(\cdot)) M v(\cdot))(x) \leq & \frac{8 \beta^{\star} K e^{-\gamma \tau}}{\pi} \sum_{k=1}^{+\infty} e^{-(2 k-1)^{2} d_{2} \tau}\left(2 K e^{-\gamma \tau-d_{2} \tau}\right)^{n-1} v(x) .
\end{aligned}
$$

Hence, we have

$$
\begin{aligned}
\sum_{n=1}^{+\infty} \Phi^{n}(\beta(M v(\cdot)) M v(\cdot))(x) & \leq \frac{8 \beta^{\star} K e^{-\gamma \tau}}{\pi} \sum_{k=1}^{+\infty} e^{-(2 k-1)^{2} d_{2} \tau} \sum_{n=1}^{+\infty}\left(2 K e^{-\gamma \tau-d_{2} \tau}\right)^{n-1} v(x) \\
& =\frac{8 \beta^{\star} K e^{-\gamma \tau}}{\pi\left(1-2 K e^{-\gamma \tau-d_{2} \tau}\right)}\left(\sum_{k=1}^{+\infty} e^{-(2 k-1)^{2} d_{2} \tau}\right) v(x) .
\end{aligned}
$$

Using Lemma 6.2, we can prove the following proposition on the existence of an upper solution of (33).

Proposition 7. Assume that (35) holds. Then, there exists a sufficiently large $M>0$ such that $\bar{N}(x)=M v(x)$ is an upper solution of (33). 
Proof. Let $\bar{N}(x):=M v(x)$, for $x \in[0, \pi]$. It is clear that $\bar{N}(0)=\bar{N}(\pi)=0$. Moreover, we have

$$
\begin{aligned}
& d_{1} \frac{d^{2} \bar{N}(x)}{d x^{2}}-(\delta+\beta(\bar{N}(x))) \bar{N}(x)+\frac{1-K}{K} \sum_{n=1}^{+\infty} \Phi^{n}(\beta(\bar{N}(\cdot)) \bar{N}(\cdot))(x) \\
& =-d_{1} M v(x)-(\delta+\beta(M v(x))) M v(x)+\frac{1-K}{K} \sum_{n=1}^{+\infty} \Phi^{n}(\beta(M v(\cdot)) M v(\cdot))(x) .
\end{aligned}
$$

Hence, using Lemma 6.2, we have

$$
\begin{aligned}
d_{1} & \frac{d^{2} \bar{N}(x)}{d x^{2}}-(\delta+\beta(\bar{N}(x))) \bar{N}(x)+\frac{1-K}{K} \sum_{n=1}^{+\infty} \Phi^{n}(\beta(\bar{N}(\cdot)) \bar{N}(\cdot))(x) \\
\leq & -d_{1} M v(x)-(\delta+\beta(M v(x))) M v(x) \\
& +\frac{1-K}{K} \frac{8 \beta^{\star} K e^{-\gamma \tau}}{\pi} \sum_{k=1}^{+\infty} e^{-(2 k-1)^{2} d_{2} \tau} \frac{1}{1-2 K e^{-\gamma \tau-d_{2} \tau}} v(x), \\
= & {\left[-d_{1}-\delta-\beta(M v(x))+\frac{8 \beta^{\star}(1-K) e^{-\gamma \tau}}{\pi M\left(1-2 K e^{-\gamma \tau-d_{2} \tau}\right)} \sum_{k=1}^{+\infty} e^{-(2 k-1)^{2} d_{2} \tau}\right] M v(x) . }
\end{aligned}
$$

Then, we can choose $M>0$ sufficiently large such that the last expression in (36) is nonpositive. Hence, $\bar{N}(x)=M v(x)$ is an upper solution of (33).

Now, we focus on the existence of positive solution $x \mapsto(N(x), u(x))$ of the stationary problem (31) (which is equivalent to (28)). As $N$ is independent on $u$, see the system (31), we can focus only on the equation

$$
\left\{\begin{aligned}
0=d_{1} \frac{d^{2} N(x)}{d x^{2}}- & (\delta+\beta(N(x))) N(x) \\
& +\frac{1-K}{K} \int_{0}^{\pi} \kappa(\tau, x, s) \beta(N(s)) N(s) d s, \\
N(0)=N(\pi)= & 0 .
\end{aligned}\right.
$$

We suppose the following additional condition,

$$
\beta_{\star}:=\inf _{s \geq 0}[\beta(s) s]^{\prime}>-\infty .
$$

We can first remark that if $\beta_{\star} \geq 0$, then we have $[\beta(s) s]^{\prime} \geq 0$ for all $s \geq 0$ (monotone case). In the case $\beta_{\star}<0$, the expression $s \mapsto[\beta(s) s]^{\prime}$ changes the 
sign over the interval $[0,+\infty)$ (non-monotone case). We put

$$
\mu:=\max \left\{0,-\beta_{\star}\right\} \geq 0 .
$$

Then, the stationary problem (37) is equivalent to the following system.

$$
\left\{\begin{array}{l}
-d_{1} \frac{d^{2} N(x)}{d x^{2}}+(\delta+\beta(0)) N(x)+\mu \frac{1-K}{K} \int_{0}^{\pi} \kappa(\tau, x, s) N(s) d s \\
=(\beta(0)-\beta(N(x))) N(x)+\frac{1-K}{K} \int_{0}^{\pi} \kappa(\tau, x, s)(\beta(N(s)) N(s)+\mu N(s)) d s \\
N(0)=N(\pi)=0 .
\end{array}\right.
$$

Let us denote by

$$
h(N):=\beta(N) N+\mu N, \quad N \in[0,+\infty) .
$$

This transformation leads to

$$
\left\{\begin{array}{l}
-d_{1} \frac{d^{2} N(x)}{d x^{2}}+(\delta+\beta(0)) N(x)+\mu \frac{1-K}{K} \int_{0}^{\pi} \kappa(\tau, x, s) N(s) d s \\
=(\beta(0)-\beta(N(x))) N(x)+\frac{1-K}{K} \int_{0}^{\pi} \kappa(\tau, x, s) h(N(s)) d s \\
N(0)=N(\pi)=0
\end{array}\right.
$$

with $h^{\prime}(N) \geq 0$ for $N \geq 0$. We consider the differential operator $\mathcal{L}$, defined on the domain $D(\Delta):=\left\{N \in C[0, \pi]: N \in C^{2}(0, \pi), N(0)=N(\pi)=0\right\}$ $(\overline{D(\Delta)}=C[0, \pi])$, by

$$
\mathcal{L}: N \in D(\Delta) \mapsto \mathcal{L} N=-d_{1} \frac{d^{2} N}{d x^{2}}+b_{1} N \in C[0, \pi]
$$

with $d_{1}, b_{1}>0$ and we define the linear operator $\mathcal{K}: C[0, \pi] \rightarrow C[0, \pi]$ by

$$
(\mathcal{K} N)(x)=b_{2} \int_{0}^{\pi} \kappa(\tau, x, y) N(y) d y
$$

with $b_{2} \geq 0$. Recall that for $v \in C[0, \pi]$, we have

$$
\left(\mathcal{L}^{-1} v\right)(x)=\int_{0}^{\pi} \mathcal{G}(x, y) v(y) d y \in D(\Delta),
$$


where $\mathcal{G}$ is the Green function corresponding to the operator $\mathcal{L}$ and given by

$$
\mathcal{G}(x, y)= \begin{cases}\frac{\sinh \left(\sqrt{\frac{b_{1}}{d_{1}}} x\right) \sinh \left(\sqrt{\frac{b_{1}}{d_{1}}}(\pi-y)\right)}{d_{1} \sqrt{\frac{b_{1}}{d_{1}}} \sinh \left(\sqrt{\frac{b_{1}}{d_{1}}} \pi\right)}, & 0 \leq x \leq y \leq \pi, \\ \frac{\sinh \left(\sqrt{\frac{b_{1}}{d_{1}}}(\pi-x)\right) \sinh \left(\sqrt{\frac{b_{1}}{d_{1}}} y\right)}{d_{1} \sqrt{\frac{b_{1}}{d_{1}}} \sinh \left(\sqrt{\frac{b_{1}}{d_{1}}} \pi\right)}, \quad 0 \leq y \leq x \leq \pi,\end{cases}
$$

where $\sinh (x)=\left(e^{x}-e^{-x}\right) / 2$. As in [29], we need the following two lemmas.

Lemma 6.3. Assume that

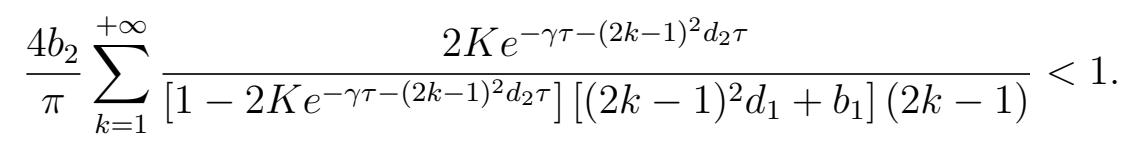

Then, the linear operator $\mathcal{L}^{-1} \mathcal{K}: C[0, \pi] \rightarrow C[0, \pi]$ satisfies $\left\|\mathcal{L}^{-1} \mathcal{K}\right\|<1$.

Proof. Let $N \in X=C[0, \pi]$. We have

$$
\begin{aligned}
& \left(\mathcal{L}^{-1} \mathcal{K} N\right)(x)=b_{2} \int_{0}^{\pi} \mathcal{G}(x, u)\left[\int_{0}^{\pi} \kappa(\tau, u, y) N(y) d y\right] d u \\
& \leq b_{2} \int_{0}^{\pi}\left[\int_{0}^{\pi} \mathcal{G}(x, u) \kappa(\tau, u, y) d u\right] d y|N|_{X} \\
& \quad=\frac{2 b_{2}}{\pi} \sum_{n=1}^{+\infty}\left(2 K e^{-\gamma \tau}\right)^{n} \sum_{k=1}^{+\infty} e^{-k^{2} d_{2} n \tau} \int_{0}^{\pi}\left[\int_{0}^{\pi} \mathcal{G}(x, u) \sin (k u) d u\right] \sin (k y) d y|N|_{X} .
\end{aligned}
$$

By using the following equality

$$
\int_{0}^{\pi} \mathcal{G}(u, x) \sin (k u) d u=\frac{1}{d_{1} k^{2}+b_{1}} \sin (k x),
$$


we obtain that

$$
\begin{aligned}
\left(\mathcal{L}^{-1} \mathcal{K} N\right)(x) & \leq \frac{2 b_{2}}{\pi} \sum_{n=1}^{+\infty}\left(2 K e^{-\gamma \tau}\right)^{n} \sum_{k=1}^{+\infty} e^{-k^{2} d_{2} n \tau} \frac{1}{k^{2} d_{1}+b_{1}} \sin (k x) \int_{0}^{\pi} \sin (k y) d y|N|_{X} \\
& =\frac{2 b_{2}}{\pi} \sum_{n=1}^{+\infty}\left(2 K e^{-\gamma \tau}\right)^{n} \sum_{k=1}^{+\infty} e^{-k^{2} d_{2} n \tau} \frac{1}{k^{2} d_{1}+b_{1}} \sin (k x) \frac{1-(-1)^{k}}{k}|N|_{X} \\
& \leq \frac{2 b_{2}}{\pi} \sum_{n=1}^{+\infty}\left(2 K e^{-\gamma \tau}\right)^{n} \sum_{k=1}^{+\infty} e^{-(2 k-1)^{2} d_{2} n \tau} \frac{2}{\left[(2 k-1)^{2} d_{1}+b_{1}\right](2 k-1)}|N|_{X} \\
& =\frac{4 b_{2}}{\pi} \sum_{k=1}^{+\infty} \sum_{n=1}^{+\infty}\left(2 K e^{-\gamma \tau-(2 k-1)^{2} d_{2} \tau}\right)^{n} \frac{1}{\left[(2 k-1)^{2} d_{1}+b_{1}\right](2 k-1)}|N|_{X} \\
& =\frac{4 b_{2}}{\pi} \sum_{k=1}^{+\infty} \frac{2 K e^{-\gamma \tau-(2 k-1)^{2} d_{2} \tau}}{\left[1-2 K e^{\left.-\gamma \tau-(2 k-1)^{2} d_{2} \tau\right]\left[(2 k-1)^{2} d_{1}+b_{1}\right](2 k-1)}\right.}|N|_{X} .
\end{aligned}
$$

Thus, by (39), we obtain

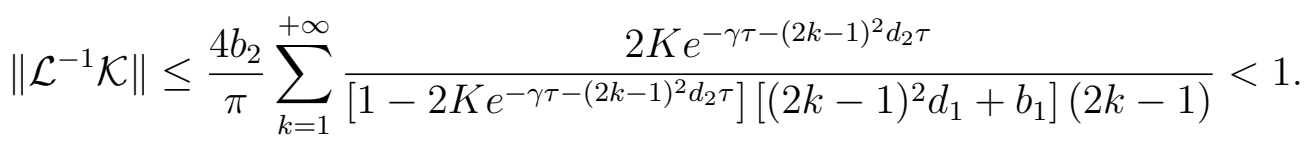

Remark 1. We remark that

$$
\frac{2 K e^{-\gamma \tau-(2 k-1)^{2} d_{2} \tau}}{\left[1-2 K e^{-\gamma \tau-(2 k-1)^{2} d_{2} \tau}\right]\left[(2 k-1)^{2} d_{1}+b_{1}\right]} \leq \frac{2 K e^{-\gamma \tau-d_{2} \tau}}{d_{1}\left(1-2 K e^{-\gamma \tau-d_{2} \tau}\right)} \frac{1}{(2 k-1)^{2}} .
$$

Then, a necessary condition to have (39) is

$$
\frac{4}{d_{1} \pi}\left(\frac{2 b_{2} K e^{-\gamma \tau-d_{2} \tau}}{1-2 K e^{-\gamma \tau-d_{2} \tau}}\right) \sum_{k=1}^{+\infty} \frac{1}{(2 k-1)^{3}}<1 .
$$

Lemma 6.4. Assume that (39) holds. Moreover, assume that

$$
\frac{4 b_{2} \sinh ^{4}\left(\frac{\sqrt{b_{1}} \pi}{2 \sqrt{d_{1}}}\right)}{\pi d_{1}\left(\sqrt{\frac{b_{1}}{d_{1}}}\right)^{3} \sinh \left(\sqrt{\frac{b_{1}}{d_{1}}} \pi\right)} \sum_{k=1}^{+\infty} \frac{2 K e^{-\gamma \tau-k^{2} d_{2} \tau}}{1-2 K e^{-\gamma \tau-k^{2} d_{2} \tau}}<1
$$

Then, the operator $(\mathcal{L}+\mathcal{K})^{-1}$ is positive. 
Proof. Using the same technique as in the proof of ([29], Lemma 4.2), under the condition $\left\|\mathcal{L}^{-1} \mathcal{K}\right\|<1$, we have

$$
(\mathcal{L}+\mathcal{K})^{-1}=\sum_{n=0}^{+\infty}\left(\mathcal{L}^{-1} \mathcal{K}\right)^{2 n}\left[\mathcal{L}^{-1}-\mathcal{L}^{-1} \mathcal{K} \mathcal{L}^{-1}\right]
$$

Then, it suffices to show that the operator $\mathcal{L}^{-1}-\mathcal{L}^{-1} \mathcal{K} \mathcal{L}^{-1}$ is positive. That means to prove that, for all $v \geq 0$, we have

$$
\mathcal{L}^{-1} v \geq \mathcal{L}^{-1} \mathcal{K} \mathcal{L}^{-1} v
$$

By Fubini's theorem, we can write

$$
\begin{aligned}
\left(\mathcal{L}^{-1} \mathcal{K} \mathcal{L}^{-1} v\right)(x) & =\int_{0}^{\pi} \mathcal{G}(x, s)\left\{b_{2} \int_{0}^{\pi} \kappa(\tau, s, u)\left[\int_{0}^{\pi} \mathcal{G}(u, y) v(y) d y\right] d u\right\} d s \\
& =\int_{0}^{\pi}\left\{\int_{0}^{\pi} \mathcal{G}(x, s)\left[b_{2} \int_{0}^{\pi} \kappa(\tau, s, u) \mathcal{G}(u, y) d u\right] d s\right\} v(y) d y
\end{aligned}
$$

Then, it is sufficient to prove that for all $x, y \in[0, \pi]$,

$$
\int_{0}^{\pi} \mathcal{G}(x, s)\left[b_{2} \int_{0}^{\pi} \kappa(\tau, s, u) \mathcal{G}(u, y) d u\right] d s \leq \mathcal{G}(x, y) .
$$

In another side, we have

$$
\begin{aligned}
\int_{0}^{\pi} \kappa(\tau, s, u) \mathcal{G}(u, y) d u & \\
= & \frac{2}{\pi} \sum_{n=1}^{+\infty}\left(2 K e^{-\gamma \tau}\right)^{n} \sum_{k=1}^{+\infty} e^{-k^{2} d_{2} n \tau}\left[\int_{0}^{\pi} \mathcal{G}(u, y) \sin (k u) d u\right] \sin (k s) .
\end{aligned}
$$

So, by using (40), we have

$$
\int_{0}^{\pi} \kappa(\tau, s, u) \mathcal{G}(u, y) d u=\frac{2}{\pi} \sum_{n=1}^{+\infty}\left(2 K e^{-\gamma \tau}\right)^{n} \sum_{k=1}^{+\infty} \frac{e^{-k^{2} d_{2} n \tau}}{d_{1} k^{2}+b_{1}} \sin (k y) \sin (k s),
$$

and

$$
\begin{aligned}
& \int_{0}^{\pi} \mathcal{G}(x, s)\left[b_{2} \int_{0}^{\pi} \kappa(\tau, s, u) \mathcal{G}(u, y) d u\right] d s \\
& =\int_{0}^{\pi} \mathcal{G}(x, s)\left[\frac{2 b_{2}}{\pi} \sum_{n=1}^{+\infty}\left(2 K e^{-\gamma \tau}\right)^{n} \sum_{k=1}^{+\infty} \frac{e^{-k^{2} d_{2} n \tau}}{d_{1} k^{2}+b_{1}} \sin (k y) \sin (k s)\right] d s \\
& =\frac{2 b_{2}}{\pi} \sum_{n=1}^{+\infty}\left(2 K e^{-\gamma \tau}\right)^{n} \sum_{k=1}^{+\infty} \frac{e^{-k^{2} d_{2} n \tau}}{\left(d_{1} k^{2}+b_{1}\right)^{2}} \sin (k y) \sin (k x) .
\end{aligned}
$$


Since,

$$
\begin{aligned}
\int_{0}^{\pi} \mathcal{G}(u, y) d u & =\int_{0}^{\pi} \mathcal{G}(y, u) d u \\
& =\frac{1}{b_{1} \sinh \sqrt{\frac{b_{1}}{d_{1}}} \pi}\left[\sinh \sqrt{\frac{b_{1}}{d_{1}}} \pi-\sinh \sqrt{\frac{b_{1}}{d_{1}}} y-\sinh \sqrt{\frac{b_{1}}{d_{1}}}(\pi-y)\right],
\end{aligned}
$$

we obtain

$$
\begin{aligned}
& \frac{1}{d_{1} k^{2}+b_{1}} \sin k y=\int_{0}^{\pi} \mathcal{G}(u, y) \sin k u d u \leq \int_{0}^{\pi} \mathcal{G}(u, y) d u \\
& =\frac{1}{b_{1} \sinh \sqrt{\frac{b_{1}}{d_{1}}} \pi}\left[\sinh \sqrt{\frac{b_{1}}{d_{1}}} \pi-\sinh \sqrt{\frac{b_{1}}{d_{1}}} y-\sinh \sqrt{\frac{b_{1}}{d_{1}}}(\pi-y)\right] .
\end{aligned}
$$

So,

$$
\begin{aligned}
\int_{0}^{\pi} \mathcal{G}(x, s) & {\left[b_{2} \int_{0}^{\pi} \kappa(\tau, s, u) \mathcal{G}(u, y) d u\right] d s } \\
& \leq \frac{2 b_{2}}{\pi} \sum_{n=1}^{+\infty}\left(2 K e^{-\gamma \tau}\right)^{n} \sum_{k=1}^{+\infty} \frac{e^{-k^{2} d_{2} n \tau}}{b_{1}^{2} \sinh ^{2} \sqrt{\frac{b_{1}}{d_{1}}} \pi} \\
& \times\left[\sinh \sqrt{\frac{b_{1}}{d_{1}}} \pi-\sinh \sqrt{\frac{b_{1}}{d_{1}}} x-\sinh \sqrt{\frac{b_{1}}{d_{1}}}(\pi-x)\right] \\
& \times\left[\sinh \sqrt{\frac{b_{1}}{d_{1}}} \pi-\sinh \sqrt{\frac{b_{1}}{d_{1}}} y-\sinh \sqrt{\frac{b_{1}}{d_{1}}}(\pi-y)\right]
\end{aligned}
$$

Consequently,

$$
\begin{aligned}
& \int_{0}^{\pi} \mathcal{G}(x, s)\left[b_{2} \int_{0}^{\pi} \kappa(\tau, s, u) \mathcal{G}(u, y) d u\right] d s \\
& \leq \frac{2 b_{2}}{\pi b_{1}^{2} \sinh ^{2} \sqrt{\frac{b_{1}}{d_{1}}} \pi}\left[\sinh \sqrt{\frac{b_{1}}{d_{1}}} \pi-\sinh \sqrt{\frac{b_{1}}{d_{1}}} x-\sinh \sqrt{\frac{b_{1}}{d_{1}}}(\pi-x)\right] \\
& \times\left[\sinh \sqrt{\frac{b_{1}}{d_{1}}} \pi-\sinh \sqrt{\frac{b_{1}}{d_{1}}} y-\sinh \sqrt{\frac{b_{1}}{d_{1}}}(\pi-y)\right] \sum_{k=1}^{+\infty} \frac{2 K e^{-\gamma \tau-k^{2} d_{2} \tau}}{1-2 K e^{-\gamma \tau-k^{2} d_{2} \tau}} .
\end{aligned}
$$


Let put

$$
c=\left(\frac{2 b_{2}}{\pi d_{1}\left(\sqrt{\frac{b_{1}}{d_{1}}}\right)^{3} \sinh \sqrt{\frac{b_{1}}{d_{1}}} \pi} \sum_{k=1}^{+\infty} \frac{2 K e^{-\gamma \tau-k^{2} d_{2} \tau}}{1-2 K e^{-\gamma \tau-k^{2} d_{2} \tau}}\right)^{\frac{1}{2}} .
$$

We can prove as in $[29]$ that, for all $x, y \in[0, \pi]$,

$$
c\left[\sinh \sqrt{\frac{b_{1}}{d_{1}}} \pi-\sinh \sqrt{\frac{b_{1}}{d_{1}}} x-\sinh \sqrt{\frac{b_{1}}{d_{1}}}(\pi-x)\right] \leq \sinh \sqrt{\frac{b_{1}}{d_{1}}} x,
$$

and

$$
c\left[\sinh \sqrt{\frac{b_{1}}{d_{1}}} \pi-\sinh \sqrt{\frac{b_{1}}{d_{1}}} x-\sinh \sqrt{\frac{b_{1}}{d_{1}}}(\pi-x)\right] \leq \sinh \sqrt{\frac{b_{1}}{d_{1}}}(\pi-x) .
$$

As a consequence, we obtain the inequality (42) and then the operator $\mathcal{L}^{-1}$ $\mathcal{L}^{-1} \mathcal{K} \mathcal{L}^{-1}$ is positive. Then, the operator $(\mathcal{L}+\mathcal{K})^{-1}$ is positive.

Remark 2. Remark that, for all $k \geq 1$, we have the inequality

$$
\frac{2 K e^{-\gamma \tau-k^{2} d_{2} \tau}}{1-2 K e^{-\gamma \tau-k^{2} d_{2} \tau}} \leq \frac{2 K e^{-\gamma \tau}}{1-2 K e^{-\gamma \tau-d_{2} \tau}}\left(e^{-d_{2} \tau}\right)^{k}
$$

Then, this implies the inequality

$$
\sum_{k=1}^{+\infty} \frac{2 K e^{-\gamma \tau-k^{2} d_{2} \tau}}{1-2 K e^{-\gamma \tau-k^{2} d_{2} \tau}} \leq \frac{2 K e^{-\gamma \tau-d_{2} \tau}}{\left(1-2 K e^{-\gamma \tau-d_{2} \tau}\right)\left(1-e^{-d_{2} \tau}\right)}
$$

Consequently, a necessary condition to have (41) is

$$
\frac{4 \sinh ^{4}\left(\frac{\sqrt{b_{1}} \pi}{2 \sqrt{d_{1}}}\right)}{\pi d_{1}\left(1-e^{-d_{2} \tau}\right)\left(\sqrt{\frac{b_{1}}{d_{1}}}\right)^{3} \sinh \sqrt{\frac{b_{1}}{d_{1}}} \pi}\left(\frac{2 b_{2} K e^{-\gamma \tau-d_{2} \tau}}{1-2 K e^{-\gamma \tau-d_{2} \tau}}\right)<1 .
$$

Using the last lemma, we prove the following theorem.

Theorem 6.5. Let $b_{1}=\delta+\beta(0)$ and $b_{2}=\mu(1-K) / K$. Assume that (35), (38), (39), (41) and $\lambda_{0}>0$ hold. Then, there exists a positive stationary solution $N(x)$ of System (37). 
Proof. From the propositions 6 and 7, the functions $\underline{N}(x)$ and $\bar{N}(x)$ are subsolution and super-solution, respectively. Let consider the operators $\mathcal{L}$ and $\mathcal{K}$ defined as before with $b_{1}=\delta+\beta(0)$ and $b_{2}=\mu(1-K) / K$. We define the nonlinear operator $\mathcal{P}: C[0, \pi] \rightarrow C^{2}(0, \pi) \cap C[0, \pi]$ by

$$
(\mathcal{P} u)(x)=(\mathcal{L}+\mathcal{K})^{-1}\left((\beta(0)-\beta(u)) u+\frac{1-K}{K} \int_{0}^{\pi} \kappa(\tau, x, s) h(u(s)) d s\right) .
$$

Recall that $u \mapsto \beta(u)$ is a decreasing function for $u \geq 0$. So, $u \mapsto(\beta(0)-$ $\beta(u)) u$ is an increasing function for $u \geq 0$. Furthermore, $u \mapsto h(u)$ is also an increasing function for $u \geq 0$. Then, we can say that $\mathcal{Q}: C[0, \pi] \rightarrow$ $C^{2}(0, \pi) \cap C[0, \pi]$ given by

$$
(\mathcal{Q} u)(x)=(\beta(0)-\beta(u)) u+\frac{1-K}{K} \int_{0}^{\pi} \kappa(\tau, x, s) h(u(s)) d s,
$$

is positive and strongly monotone. Moreover, $(\mathcal{L}+\mathcal{K})^{-1}$ is positive (see lemma 6.4). This implies that $\mathcal{P}$ is positive and strongly monotone. Then, $\mathcal{P}$ is strongly increasing on the interval $[\hat{0}, \hat{M}]$, where $\hat{M}$ (respectively, $\hat{0}$ ) denotes the constant function on $[0, \pi]$ taking the value $M$ (respectively, 0 ) and $M$ is the constant given by the proposition 7. By using a standard argument of sub- and super-solutions (see [32]) and the propositions 6 and 7, we conclude that there exists a positive stationary solution $N^{\star}(x)$ of System (37), which satisfies

$$
0<\underline{N}(x) \leq N^{\star}(x) \leq \bar{N}(x), \quad \text { for all } \quad(0, \pi) .
$$

Remark 3. The conditions (39) and (41) can hold simultaneously. To see that, we first remark that if $\beta_{\star} \geq 0$, then we have $\mu=0$ (the monotone case). In this case, (39) and (41) are always satisfied. For the non-monotone case, $\beta_{\star}<0$, we can choose $\mu=-\beta_{\star}$ small enough to have (39) and (41).

For the uniqueness of the stationary solution of problem (37), we make the following additional assumption.

$$
s \mapsto\left[\left(\frac{2 e^{-\gamma \tau-d_{2} \tau}-1}{1-2 K e^{-\gamma \tau-d_{2} \tau}}\right) \beta(s)-\left(d_{1}+\delta\right)\right] s \text { is nondecreasing for } s \geq 0 .
$$

Under the assumption (43), we prove the following theorem on the uniqueness of the stationary solution. 
Theorem 6.6. Suppose that the assumptions of Theorem 6.5 and (43) hold. Then, problem (37) has at most one positive stationary solution.

Proof. The proof of this result is similar to the proof of the corresponding result in [20].

\section{Persistence}

In this section, we investigate weak persistence and persistence of the system (6), for $\lambda_{0}>0$. Let us consider an arbitrary large positive constant $M>0$ and define the set

$$
\begin{aligned}
& D_{M}:=\left\{(\phi, \psi) \in X^{+} \times C^{+}: \phi(x)>0 \text { for some } x \in(0, \pi),\right. \\
&\psi(\theta)(x)=\psi(\theta, x) \leq M v(x) \text { for all }(\theta, x) \in[-\tau, 0] \times[0, \pi]\},
\end{aligned}
$$

where $v(x)=\sin x$. The system (6) can be written as follows

$$
\left\{\begin{array}{l}
\frac{\partial N}{d t}(t, x)=d_{1} \frac{\partial^{2} N}{\partial x^{2}}(t, x)-\left(\delta+\frac{1}{K} \beta(N(t, x))\right) N(t, x)+\frac{1-K}{K} u(t, x), \\
u(t, x)=\beta(N(t, x)) N(t, x)+2 K e^{-\gamma \tau} \int_{0}^{\pi} \Gamma_{2}(\tau, x, y) u(t-\tau, y) d y \\
N(t, 0)=N(t, \pi)=u(t, 0)=u(t, \pi)=0 \\
N(0, x)=N_{0}(x), \\
u(\theta, x)=u_{0}(\theta, x), \quad \theta \in[-\tau, 0] .
\end{array}\right.
$$

We first prove the following lemma on the boundedness of solutions.

Lemma 7.1. Assume that (35) holds and $\left(N_{0}, u_{0}\right) \in D_{M}$. Then, there exist positive uniform upper bounds $N^{+}>0$ and $u^{+}>0$ such that $N(t, x) \leq N^{+}$ and $u(t, x) \leq u^{+}$, for all $t>0$ and $x \in[0, \pi]$.

Proof. For any $t>0$, let $n:=\lfloor t / \tau\rfloor$ be the integer part of $t / \tau$. We have

$$
\begin{aligned}
u(t, x) & =\beta(N(t, x)) N(t, x)+2 K e^{-\gamma \tau} \int_{0}^{\pi} \Gamma_{2}(\tau, x, y) u(t-\tau, y) d y \\
& =\sum_{k=0}^{n} \Phi^{k}(\beta(N(t-k \tau, \cdot)) N(t-k \tau, \cdot))(x)+\Phi^{n+1}\left(u_{0}(t-(n+1) \tau, \cdot)\right)(x), \\
& \leq \sum_{k=0}^{n} \Phi^{k}\left(\beta^{\star} \mathbf{1}(\cdot)\right)(x)+M \Phi^{n+1}(v(\cdot))(x),
\end{aligned}
$$


where $\Phi$ is the linear operator defined by (29), and $\mathbf{1} \in X$ denotes the constant function taking the value 1 for any $x \in[0, \pi]$. Recalling that $\Phi(v)=$ $2 K e^{-\gamma \tau-d_{2} \tau} v$ and $\sum_{k=0}^{+\infty} \Phi^{k}=(I d-\Phi)^{-1}$. Thus, we have

$$
\begin{aligned}
u(t, x) & \leq \beta^{\star} \sum_{k=0}^{+\infty} \Phi^{k}(\mathbf{1}(\cdot))(x)+M\left(2 K e^{-\gamma \tau-d_{2} \tau}\right)^{n+1} v(x), \\
& \leq \beta^{*}(I d-\Phi)^{-1} \mathbf{1}(x)+M
\end{aligned}
$$

Hence, for $u^{+}:=\sup _{x \in[0, \pi]}\left(\beta^{*}(I d-\Phi)^{-1} \mathbf{1}(x)+M\right) \in(0,+\infty)$, we obtain $u(t, x) \leq u^{+}$, for all $t>0$ and $x \in[0, \pi]$.

Let $T>0$ be a positive constant. From the first equation of (44), we have for all $t>T$ and $x \in[0, \pi]$ that

$$
\begin{aligned}
N(t, x) & \leq T_{1}(t) N_{0}(x)+\frac{1-K}{K} \int_{0}^{t} T_{1}(t-s) u(s, \cdot)(x) d s \\
= & \int_{0}^{\pi} \Gamma_{1}(t, x, y) N_{0}(y) d y+\frac{1-K}{K} \int_{0}^{t} \int_{0}^{\pi} \Gamma_{1}(t-s, x, y) u(s, y) d y d s \\
& \leq \frac{2}{\pi\left(e^{d_{1} t}-1\right)} \int_{0}^{\pi} N_{0}(y) d y+\frac{1-K}{K} \int_{0}^{t} \int_{0}^{\pi} \frac{2}{\pi} \sum_{k \geq 1} e^{-k^{2} d_{1}(t-s)} \sin k x \sin k y d y d s u^{+}, \\
& \leq \frac{2}{\pi\left(e^{d_{1} T}-1\right)} \int_{0}^{\pi} N_{0}(y) d y+\frac{1-K}{K} \frac{2 u^{+}}{\pi} \sum_{k \geq 1} \frac{1}{k^{2} d_{1}}\left(1-e^{-k^{2} d_{1} t}\right) \int_{0}^{\pi} \sin k x \sin k y d y \\
& \leq \frac{2}{\pi\left(e^{d_{1} T}-1\right)} \int_{0}^{\pi} N_{0}(y) d y+\frac{1-K}{K} 2 u^{+} \sum_{k \geq 1} \frac{1}{k^{2} d_{1}}=: N_{1}<+\infty .
\end{aligned}
$$

Hence, setting $N^{+}:=\max \left\{N_{1}, \sup _{(t, x) \in[0, T] \times[0, \pi]} N(t, x)\right\}$, the proof is complete.

Using Lemma 7.1, we prove the following lemma on the weak persistence of the system (44).

Lemma 7.2. Assume that (35) holds and $\lambda_{0}>0$. Then, for any $\left(N_{0}, u_{0}\right) \in$ $D_{M}$, there exists a positive constant $\epsilon>0$ such that

$$
\begin{aligned}
& \limsup _{t \rightarrow+\infty}|N(t, \cdot)|_{X}>\epsilon, \\
& \limsup _{t \rightarrow+\infty}|u(t, \cdot)|_{X}>\epsilon .
\end{aligned}
$$


Proof. Note that $\lambda_{0}$ can be written as follows.

$$
\lambda_{0}=-d_{1}-\delta-\frac{1}{K} \beta(0)+\frac{1-K}{K} \frac{\beta(0)}{1-2 K e^{-\gamma \tau-d_{2} \tau}} .
$$

Since $\lambda_{0}>0$, one can choose $\epsilon>0$ sufficiently small and $\lambda>0$ such that

$$
-\left(\lambda+d_{1}+\delta+\frac{1}{K} \beta(0)\right)+\frac{1-K}{K} \frac{\beta(\epsilon)}{1-2 K e^{-\gamma \tau-d_{2} \tau-\lambda \tau}}>0 .
$$

Suppose by contradiction that (45) does not hold. Then, there exists a sufficiently large $T>0$ such that $N(t, x) \leq \epsilon$, for all $t \geq T$ and $x \in[0, \pi]$. From the first equation of (44), we have

$$
\begin{aligned}
& \int_{0}^{\pi} v(x) \frac{\partial N(t, x)}{\partial t} d x \\
& =d_{1} \int_{0}^{\pi} v(x) \frac{\partial^{2} N(t, x)}{\partial x^{2}}-\int_{0}^{\pi} v(x)\left(\delta+\frac{1}{K} \beta(N(t, x))\right) N(t, x) d x \\
& \quad+\frac{1-K}{K} \int_{0}^{\pi} v(x) u(t, x) d x \\
& \geq-\left(d_{1}+\delta+\frac{1}{K} \beta(0)\right) \int_{0}^{\pi} v(x) N(t, x) d x+\frac{1-K}{K} \int_{0}^{\pi} v(x) u(t, x) d x .
\end{aligned}
$$

Furthermore, from the second equation of (44), we have

$$
\begin{aligned}
& \int_{0}^{\pi} v(x) u(t, x) d x \\
= & \int_{0}^{\pi} v(x) \beta(N(t, x)) N(t, x) d x \\
& \quad+2 K e^{-\gamma \tau} \int_{0}^{\pi} v(x) \int_{0}^{\pi} \Gamma_{2}(\tau, x, y) u(t-\tau, y) d y d x, \\
= & \int_{0}^{\pi} v(x) \beta(N(t, x)) N(t, x) d x+2 K e^{-\gamma \tau-d_{2} \tau} \int_{0}^{\pi} v(x) u(t-\tau, x) d x .
\end{aligned}
$$

Multiplying (49) by $e^{-\lambda t}$ and integrating from $t=T$ to $t=+\infty$, we obtain

$$
\begin{array}{r}
\int_{T}^{+\infty} e^{-\lambda t} \int_{0}^{\pi} v(x) u(t, x) d x d t=\int_{T}^{+\infty} e^{-\lambda t} \int_{0}^{\pi} v(x) \beta(N(t, x)) N(t, x) d x d t \\
+2 K e^{-\gamma \tau-d_{2} \tau} \int_{T}^{+\infty} e^{-\lambda t} \int_{0}^{\pi} v(x) u(t-\tau, x) d x d t
\end{array}
$$


Then, we obtain

$$
\begin{aligned}
\int_{T}^{+\infty} & e^{-\lambda t} \int_{0}^{\pi} v(x) u(t, x) d x d t \\
\geq & \beta(\epsilon) \int_{T}^{+\infty} e^{-\lambda t} \int_{0}^{\pi} v(x) N(t, x) d x d t \\
& +2 K e^{-\gamma \tau-d_{2} \tau-\lambda \tau} \int_{T}^{+\infty} e^{-\lambda(t-\tau)} \int_{0}^{\pi} v(x) u(t-\tau, x) d x d t \\
= & \beta(\epsilon) \int_{T}^{+\infty} e^{-\lambda t} \int_{0}^{\pi} v(x) N(t, x) d x d t \\
& +2 K e^{-\gamma \tau-d_{2} \tau-\lambda \tau} \int_{T-\tau}^{+\infty} e^{-\lambda t} \int_{0}^{\pi} v(x) u(t, x) d x d t \\
\geq & \beta(\epsilon) \int_{T}^{+\infty} e^{-\lambda t} \int_{0}^{\pi} v(x) N(t, x) d x d t \\
& +2 K e^{-\gamma \tau-d_{2} \tau-\lambda \tau} \int_{T}^{+\infty} e^{-\lambda t} \int_{0}^{\pi} v(x) u(t, x) d x d t
\end{aligned}
$$

Hence, we have

$$
\int_{T}^{+\infty} e^{-\lambda t} \int_{0}^{\pi} v(x) u(t, x) d x d t \geq \frac{\beta(\epsilon)}{1-2 K e^{-\gamma \tau-d_{2} \tau-\lambda \tau}} \int_{T}^{+\infty} e^{-\lambda t} \int_{0}^{\pi} v(x) N(t, x) d x d t .
$$

On the other hand, multiplying (48) by $e^{-\lambda t}$ and integration from $t=T$ to $t=+\infty$, we obtain

$$
\begin{aligned}
\int_{T}^{+\infty} & e^{-\lambda t} \int_{0}^{\pi} v(x) \frac{\partial N(t, x)}{\partial t} d x d t \\
= & -e^{-\lambda T} \int_{0}^{\pi} v(x) N(T, x) d x+\lambda \int_{T}^{+\infty} e^{-\lambda t} \int_{0}^{\pi} v(x) N(t, x) d x d t \\
\geq & -\left(d_{1}+\delta+\frac{1}{K} \beta(0)\right) \int_{T}^{+\infty} e^{-\lambda t} \int_{0}^{\pi} v(x) N(t, x) d x d t \\
& +\frac{1-K}{K} \int_{T}^{+\infty} e^{-\lambda t} \int_{0}^{\pi} v(x) u(t, x) d x d t
\end{aligned}
$$


Hence, from (47), (50) and (51), we have

$$
\begin{aligned}
0> & -e^{-\lambda T} \int_{0}^{\pi} v(x) N(T, x) d x \\
\geq & {\left[-\left(\lambda+d_{1}+\delta+\frac{1}{K} \beta(0)\right)+\frac{1-K}{K} \frac{\beta(\epsilon)}{1-2 K e^{-\gamma \tau-d_{2} \tau-\lambda \tau}}\right] } \\
& \times \int_{T}^{+\infty} e^{-\lambda t} \int_{0}^{\pi} v(x) N(t, x) d x d t \\
> & 0 .
\end{aligned}
$$

This gives a contradiction. Then, we conclude that (45) is true.

Next we show the inequality (46). From the second equation of (6), we have

$$
u(t, x)>\beta(N(t, x)) N(t, x),
$$

and from Lemma 7.1, we have

$$
u(t, x)>\beta\left(N^{+}\right) N(t, x),
$$

where $N^{+}$denotes the uniform upper bound of $N$. Hence, from (45), we obtain

$$
\limsup _{t \rightarrow+\infty}|u(t, \cdot)|_{X}>\beta\left(N^{+}\right) \epsilon .
$$

Setting $\min \left(\epsilon, \beta\left(N^{+}\right) \epsilon\right)$ to be $\epsilon$ again, the proof is complete.

Note that we did not use Lemma 7.1 to proof (45). Therefore, we have the following corollary.

Corollary 1. Assume that $\lambda_{0}>0$. Then, for any $\left(N_{0}, u_{0}\right) \in X^{+} \times C^{+}$such that $N_{0}(x)>0$, for some $x \in(0, \pi)$, there exists a positive constant $\epsilon>0$ such that

$$
\limsup _{t \rightarrow+\infty}|N(t, \cdot)|_{X}>\epsilon
$$

Finally, based on Lemma 7.2 and Corollary 1, we prove the persistence of System (6). We need the following additional condition

$$
2 K e^{-\gamma \tau} \sup _{x \in[0, \pi]} \int_{0}^{\pi} \Gamma_{2}(\tau, x, y) d y<1 .
$$

One can remark that the condition (52) is stronger than the condition (16). 
Theorem 7.3. Assume that (35), (52) hold and $\lambda_{0}>0$. Then, for any $\left(N_{0}, u_{0}\right) \in D_{M}$, there exists a positive constant $\epsilon_{0}>0$ such that

$$
\begin{aligned}
& \liminf _{t \rightarrow+\infty}|N(t, \cdot)|_{X}>\epsilon_{0}, \\
& \liminf _{t \rightarrow+\infty}|u(t, \cdot)|_{X}>\epsilon_{0} .
\end{aligned}
$$

Proof. We first prove (53). From Corollary 1, there exists an increasing sequence $\left\{\tau_{k}\right\}_{k=1}^{+\infty}>0$, such that

$$
\left|N\left(\tau_{k}, \cdot\right)\right|_{X}>\epsilon .
$$

Suppose that (53) does not hold. Then, there exist an increasing sequence $\left\{t_{k}\right\}_{k=1}^{+\infty}$ and a decreasing sequence $\left\{e_{k}\right\}_{k=1}^{+\infty}$ such that $t_{k}>\tau_{k}, \lim _{k \rightarrow+\infty} e_{k}=0$ and

$$
\left|N\left(t_{k}, \cdot\right)\right|_{X}<e_{k}<\epsilon .
$$

From the continuity, there exists a sequence $\left\{\theta_{k}\right\}_{k=1}^{+\infty}$ such that $\theta_{k} \in\left(\tau_{k}, t_{k}\right)$ and

$$
\left|N\left(\theta_{k}, \cdot\right)\right|_{X}=\epsilon \text { and }|N(t, \cdot)|_{X}<\epsilon \text { for all } t \in\left(\theta_{k}, t_{k}\right) .
$$

Let $\left\{N_{k}\right\}_{k=1}^{+\infty}$ be a functional sequence in $X$ such that $N_{k}:=N\left(\theta_{k}, \cdot\right) \in X$. Since

$$
\left|N_{k}(x)\right| \leq\left|N_{k}\right|_{X}=\left|N\left(\theta_{k}, \cdot\right)\right|_{X}=\epsilon \text {, for all } x \in[0, \pi],
$$

the sequence $\left\{N_{k}\right\}_{k=1}^{+\infty}$ is uniformly bounded. For $x, x^{\prime} \in[0, \pi]$, we have from 
the first equality of (44) and Lemma 7.1, that

$$
\begin{aligned}
\mid & N_{k}(x)-N_{k}\left(x^{\prime}\right)\left|\leq \int_{0}^{\pi}\right| \Gamma_{1}\left(\theta_{k}, x, y\right)-\Gamma_{1}\left(\theta_{k}, x^{\prime}, y\right) \mid N_{0}(y) d y \\
& +\int_{0}^{\theta_{k}} \int_{0}^{\pi}\left|\Gamma_{1}\left(\theta_{k}-s, x, y\right)-\Gamma_{1}\left(\theta_{k}-s, x^{\prime}, y\right)\right|\left(\delta+\frac{1}{K} \beta(N(s, y))\right) N(s, y) d y d s \\
& +\frac{1-K}{K} \int_{0}^{\theta_{k}} \int_{0}^{\pi}\left|\Gamma_{1}\left(\theta_{k}-s, x, y\right)-\Gamma_{1}\left(\theta_{k}-s, x^{\prime}, y\right)\right| u(s, y) d y d s, \\
\leq & \int_{0}^{\pi} \frac{2}{\pi} \sum_{\ell \geq 1} e^{-\ell^{2} d_{1} \theta_{k}}\left|\sin \ell x-\sin \ell x^{\prime}\right||\sin \ell y| N_{0}(y) d y \\
& +\int_{0}^{\theta_{k}} \int_{0}^{\pi} \frac{2}{\pi} \sum_{\ell \geq 1} e^{-\ell^{2} d_{1}\left(\theta_{k}-s\right)}\left|\sin \ell x-\sin \ell x^{\prime}\right||\sin \ell y| d y d s\left(\delta+\frac{1}{K} \beta(0)\right) N^{+} \\
& +\frac{1-K}{K} \int_{0}^{\theta_{k}} \int_{0}^{\pi} \frac{2}{\pi} \sum_{\ell \geq 1} e^{-\ell^{2} d_{1}\left(\theta_{k}-s\right)}\left|\sin \ell x-\sin \ell x^{\prime}\right||\sin \ell y| d y d s u^{+}, \\
\leq & \frac{2}{\pi}\left(\int_{0}^{\pi} N_{0}(y) d y\right) \sum_{\ell \geq 1} e^{-\ell^{2} d_{1} \theta_{1}}\left|\sin \ell x-\sin \ell x^{\prime}\right| \\
& +\frac{2}{d_{1}}\left\{\left(\delta+\frac{1}{K} \beta(0)\right) N^{+}+\frac{1-K}{K} u^{+}\right\} \sum_{\ell \geq 1} \frac{1}{\ell^{2}}\left|\sin \ell x-\sin \ell x^{\prime}\right| .
\end{aligned}
$$

As the functions $x \mapsto \sum_{\ell \geq 1} e^{-\ell^{2} d_{1} \theta_{1}} \sin \ell x$ and $x \mapsto \sum_{\ell \geq 1} \frac{1}{\ell^{2}} \sin \ell x$ are uniformly continuous, the inequality (57) implies that the sequence $\left\{N_{k}\right\}_{k=1}^{+\infty}$ is equicontinuous. It follows from the Ascoli-Arzela theorem the existence of $N^{*} \in X^{+}$such that $\lim _{k \rightarrow+\infty} N_{k}=N^{*}$ (otherwise, we can choose a convergent subsequence). Let us consider the following problem

$v(t, x)= \begin{cases}\beta\left(N^{*}(x)\right) N^{*}(x)+2 K e^{-\gamma \tau} \int_{0}^{\pi} \Gamma_{2}(\tau, x, y) v(t-\tau, y) d y, & t>0, \\ u_{0}(t, x), & t \in[-\tau, 0] .\end{cases}$

For each initial condition $u_{0} \in C:=C([-\tau, 0], X)=C([-\tau, 0] \times[0, \pi], \mathbb{R})$, the difference equation (58) has a unique solution $v$, which is continuous on $(0,+\infty) \times[0, \pi]$. Let $\left\{u_{k}\right\}_{k=1}^{+\infty}$ be a functional sequence in $C$ such that $u_{k}(h, x):=v\left(\theta_{k}+h, x\right),(h, x) \in[-\tau, 0] \times[0, \pi]$, with $\theta_{k}>\tau$, for $k$ large enough. Then,

$$
u_{k}(h, x)=\beta\left(N^{*}(x)\right) N^{*}(x)+2 K e^{-\gamma \tau} \int_{0}^{\pi} \Gamma_{2}(\tau, x, y) u_{k}(h-\tau, y) d y .
$$


From Lemma 7.1, $u_{k} \leq u^{+}$and thus, the uniform boundedness follows. For $(h, x),\left(h^{\prime}, x^{\prime}\right) \in[-\tau, 0] \times[0, \pi]$, we have

$$
\left|u_{k}(h, x)-u_{k}\left(h^{\prime}, x^{\prime}\right)\right| \leq\left|u_{k}(h, x)-u_{k}\left(h, x^{\prime}\right)\right|+\left|u_{k}\left(h, x^{\prime}\right)-u_{k}\left(h^{\prime}, x^{\prime}\right)\right| .
$$

For the first term in the right-hand side of (59), we have

$$
\begin{aligned}
\left|u_{k}(h, x)-u_{k}\left(h, x^{\prime}\right)\right| \leq & \left|\beta\left(N^{*}(x)\right) N^{*}(x)-\beta\left(N^{*}\left(x^{\prime}\right)\right) N^{*}\left(x^{\prime}\right)\right| \\
& +2 K e^{-\gamma \tau} \int_{0}^{\pi}\left|\Gamma_{2}(\tau, x, y)-\Gamma_{2}\left(\tau, x^{\prime}, y\right)\right| d y u^{+} .
\end{aligned}
$$

For the second term in the right-hand side of (59), we have from (52) that

$$
\begin{aligned}
& \left|u_{k}\left(h, x^{\prime}\right)-u_{k}\left(h^{\prime}, x^{\prime}\right)\right| \leq\left|u_{k}(h, \cdot)-u_{k}\left(h^{\prime}, \cdot\right)\right|_{X} \\
& \leq 2 K e^{-\gamma \tau} \sup _{x^{\prime} \in[0, \pi]} \int_{0}^{\pi} \Gamma_{2}\left(\tau, x^{\prime}, y\right)\left|u_{k}(h-\tau, y)-u_{k}\left(h^{\prime}-\tau, y\right)\right| d y \\
& \leq 2 K e^{-\gamma \tau} \sup _{x^{\prime} \in[0, \pi]} \int_{0}^{\pi} \Gamma_{2}\left(\tau, x^{\prime}, y\right) d y\left|u_{k}(h-\tau, \cdot)-u_{k}\left(h^{\prime}-\tau, \cdot\right)\right|_{X} \\
& \leq\left(2 K e^{-\gamma \tau} \sup _{x^{\prime} \in[0, \pi]} \int_{0}^{\pi} \Gamma_{2}\left(\tau, x^{\prime}, y\right) d y\right)^{n_{k}+1} \\
& \quad \quad \times\left|u_{0}\left(\theta_{k}+h-\left(n_{k}+1\right) \tau, \cdot\right)-u_{0}\left(\theta_{k}+h^{\prime}-\left(n_{k}+1\right), \cdot\right)\right|_{X} \\
& \leq\left|u_{0}\left(\theta_{k}+h-\left(n_{k}+1\right) \tau, \cdot\right)-u_{0}\left(\theta_{k}+h^{\prime}-\left(n_{k}+1\right), \cdot\right)\right|_{X}
\end{aligned}
$$

where $n_{k}:=\left\lfloor\theta_{k} / \tau\right\rfloor$. Since $u_{0}$ is uniformly continuous on $[-\tau, 0] \times[0, \pi]$, the inequalities (59)-(60) imply that the sequence $\left\{u_{k}\right\}_{k=1}^{+\infty}$ is equicontinuous. Hence, it follows from the Ascoli-Arzela theorem that there exists $u^{*} \in C^{+}$ such that $\lim _{k \rightarrow+\infty} u_{k}=u^{*}$ (otherwise we can choose a convergent subsequence).

Let $\tilde{N}$ be a solution of (44) for an initial condition $\left(N^{*}, u^{*}\right) \in X^{+} \times C^{+}$. One can remark that $\left(N^{*}, u^{*}\right)$ is not necessary a steady state. From Corollary 1 , there exist some $\tau^{\prime}>0$ and $m>0$ such that

$$
\left|\tilde{N}\left(\tau^{\prime}, \cdot\right)\right|_{X}>\epsilon \text { and }|\tilde{N}(t, \cdot)|_{X}>m, \text { for all } t \in\left(0, \tau^{\prime}\right) .
$$

For each $k \in \mathbb{N}$, let $\tilde{N}_{k}(t, \cdot):=N\left(\theta_{k}+t, \cdot\right)$. From (61) and the continuity, for sufficiently large $k$, we have

$$
\left|\tilde{N}_{k}\left(\tau^{\prime}, \cdot\right)\right|_{X}>\epsilon \text { and }\left|\tilde{N}_{k}(t, \cdot)\right|_{X}>m>e_{k}, \text { for all } t \in\left(0, \tau^{\prime}\right) .
$$


On the other hand, for $\tilde{t}_{k}:=t_{k}-\theta_{k}>0$, we have from (55) and (56) that

$$
\left|\tilde{N}_{k}\left(\tilde{t}_{k}, \cdot\right)\right|_{X}<e_{k}<\epsilon \text { and }\left|\tilde{N}_{k}(t, \cdot)\right|_{X}<\epsilon \text {, for all } t \in\left(0, \tilde{t}_{k}\right) \text {. }
$$

If $\tau^{\prime}<\tilde{t}_{k}$, then the second inequality in (63) contradicts the first inequality in (62). If $\tau^{\prime} \geq \tilde{t}_{k}$, then the first inequality in (63) contradicts the second inequality in (62). Consequently, (53) holds. As shown in the proof of Lemma 7.1 and 7.2 , we have

$$
u(t, x)>\beta\left(N^{+}\right) N(t, x) .
$$

Hence, (54) directly follows from (53). This completes the proof.

\section{Conclusion}

We constructed a mathematical model of HSC population taking into account the spatial distribution of cells in the bone marrow, quiescent and proliferating phase with long- and short-term proliferation. The resulting model consists of a coupled system of reaction-diffusion equation and difference one with a nonlocal spatial term and time delay. We considered a bounded space with Dirichlet boundary conditions. The mathematical analysis first stressed, based on the construction of lower and upper solutions and the use of comparison principle, the basic properties of the solutions, such as existence, uniqueness, positivity and boundedness. We then studied the stability of the two steady states of the model. A sufficient condition for the global asymptotic stability of the trivial steady state, corresponding to the cells' dying out, was obtained using a Lyapunov function. By using the method of lower and upper solutions, we also obtained a sufficient condition for the existence and uniqueness of positive steady state. Finally, we investigated when the trivial steady state is unstable, the persistence of the solutions of the system.

It is believed that many hematological diseases are due to some abnormalities in the feedback loops between different compartments of HSC populations $[13,15,33]$. Among a wide variety of disorders affecting blood cells, myeloproliferative diseases are of great interest. They are characterized by a group of conditions that cause blood cells to grow abnormally. The excessive proliferation of malignant HSCs changes normal cell distribution in the bone marrow and the disease can invade the whole bone marrow. The model developed in this paper may be helpful in understanding the uncontrolled proliferation of blood cells in these hematological disorders. 


\section{References}

[1] J. P. Matson, J. G. Cook, Cell cycle proliferation decisions: the impact of single cell analyses, The FEBS Journal 284 (3) (2017) 362-375. doi:10.1111/febs.13898.

URL https ://febs.onlinelibrary.wiley.com/doi/abs/10.1111/febs.13898

[2] M. C. Mackey, Cell kinetic status of haematopoietic stem cells, Cell Proliferation 34 (2) (2001) 71-83. arXiv:https://onlinelibrary.wiley.com/doi/pdf/10.1046/j.13652184.2001.00195.x, doi:10.1046/j.1365-2184.2001.00195.x. URL https://onlinelibrary.wiley.com/doi/abs/10.1046/j.1365-2184.2001.00195.x

[3] L. Pujo-Menjouet, S. Bernard, M. Mackey, Long period oscillations in a $\mathrm{G}_{0}$ model of hematopoietic stem cells, SIAM J. Appl. Dyn. Syst. 4 (2) (2005) 312-332. doi:10.1137/030600473.

URL http://epubs.siam.org/doi/abs/10.1137/030600473

[4] S. H. Cheshier, S. J. Morrison, X. Liao, I. L. Weissman, In vivo proliferation and cell cycle kinetics of long-term self-renewing hematopoietic stem cells, Proceedings of the National Academy of Sciences 96 (6) (1999) 3120-3125. arXiv:https://www.pnas.org/content/96/6/3120.full.pdf, doi:10.1073/pnas.96.6.3120.

URL https://www . pnas .org/content/96/6/3120

[5] F. J. Burns, I. F. Tannock, On the existence of a $\mathrm{G}_{0}$-phase in the cell cycle, Cell Proliferation 3 (4) (1970) 321-334.

[6] F. Ficara, M. J. Murphy, M. Lin, M. L. Cleary, Pbx1 regulates self-renewal of long-term hematopoietic stem cells by maintaining their quiescence, Cell Stem Cell 2 (5) (2008) 484-496. doi:10.1016/j.stem.2008.03.004.

[7] P. Vegh, J. Winckler, F. Melchers, Long-term in vitro proliferating mouse hematopoietic progenitor cell lines, Immunology Letters 130 (12) (2010) 32-35. doi:10.1016/j.imlet.2010.02.001.

URL http://www.sciencedirect.com/science/article/pii/S0165247810000544

[8] A. Wilson, E. Laurenti, G. Oser, R. C. van der Wath, W. Blanco-Bose, M. Jaworski, S. Offner, C. F. Dunant, L. Eshkind, E. Bockamp, P. Li, 
H. R. MacDonald, A. Trumpp, Hematopoietic Stem Cells Reversibly Switch from Dormancy to Self-Renewal during Homeostasis and Repair, Cell 135 (6) (2008) 1118-1129. doi:10.1016/j.cell.2008.10.048.

URL http://www. sciencedirect.com/science/article/pii/S009286740801386X

[9] M. C. Mackey, Unified hypothesis for the origin of aplastic anemia and periodic hematopoiesis, Blood 51 (5) (1978) 941-956. URL http://www.bloodjournal .org/content/51/5/941

[10] M. C. Mackey, Dynamic haematological disorders of stem cell origin, Biophysical and Biochemical Information Transfer in Recognition.

[11] M. Adimy, F. Crauste, S. Ruan, A mathematical study of the hematopoiesis process with applications to chronic myelogenous leukemia, SIAM J. Appl. Math. 65 (4) (2005) 1328-1352. doi: $10.1137 / 040604698$.

URL http://epubs.siam.org/doi/abs/10.1137/040604698

[12] M. Adimy, F. Crauste, S. Ruan, Stability and Hopf bifurcation in a mathematical model of pluripotent stem cell dynamics, Nonlinear Analysis: Real World Applications 6 (4) (2005) 651-670. doi:10.1016/j.nonrwa.2004.12.010.

URL http://www. sciencedirect.com/science/article/pii/S1468121804001208

[13] S. Bernard, J. Bélair, M. C. Mackey, Oscillations in cyclical neutropenia: new evidence based on mathematical modeling, Journal of Theoretical Biology 223 (3) (2003) 283-298. doi:10.1016/S0022-5193(03)00090-0. URL http://www. sciencedirect. com/science/article/pii/S0022519303000900

[14] S. Bernard, J. Bélair, M. C. Mackey, Bifurcations in a white-blood-cell production model, Comptes Rendus Biologies 327 (3) (2004) 201-210. doi:10.1016/j.crvi.2003.05.005.

URL http://www.sciencedirect.com/science/article/pii/S1631069104000381

[15] C. Colijn, M. C. Mackey, A mathematical model of hematopoiesis -I. Periodic chronic myelogenous leukemia, Journal of Theoretical Biology 237 (2) (2005) 117-132. doi:10.1016/j.jtbi.2005.03.033. URL http://www.sciencedirect.com/science/article/pii/S0022519305001542

[16] M. C. Mackey, C. Ou, L. Pujo-Menjouet, J. Wu, Periodic oscillations of blood cell populations in chronic myelogenous leukemia, SIAM J. Math. 
Anal. 38 (1) (2006) 166-187. doi:10.1137/04061578X.

URL http://epubs.siam.org/doi/abs/10.1137/04061578X

[17] L. Pujo-Menjouet, M. C. Mackey, Contribution to the study of periodic chronic myelogenous leukemia, Comptes Rendus Biologies 327 (3) (2004) 235-244. doi:10.1016/j.crvi.2003.05.004.

URL http://www.sciencedirect.com/science/article/pii/S1631069104000253

[18] M. Adimy, A. Chekroun, T.-M. Touaoula, Age-structured and delay differential-difference model of hematopoietic stem cell dynamics, Discrete and Continuous Dynamical Systems - Series B 20 (9) (2015) 2765-2791. doi:10.3934/dcdsb.2015.20.2765.

URL http://www . aimsciences . org/journals/displayArticlesnew . jsp?paperID=11643

[19] O. Diekmann, P. Getto, Y. Nakata, On the characteristic equation $\lambda=\alpha_{1}+\left(\alpha_{2}+\alpha_{3} \lambda\right) e^{-\lambda}$ and its use in the context of a cell population model, Journal of Mathematical Biology 72 (4) (2016) 877-908. doi:10.1007/s00285-015-0918-8. URL https://doi.org/10.1007/s00285-015-0918-8

[20] M. Adimy, A. Chekroun, T. Kuniya, Delayed nonlocal reaction-diffusion model for hematopoietic stem cell dynamics with Dirichlet boundary conditions, Mathematical Modelling of Natural Phenomena 12 (6) (2017) 1-22. doi:10.1051/mmnp/2017078. URL https:/articles/mmnp/abs/2017/06/mmnp170078s/mmnp170078s.html

[21] N. Bessonov, F. Crauste, I. Demin, V. Volpert, Dynamics of erythroid progenitors and erythroleukemia, Mathematical Modelling of Natural Phenomena 4 (3) (2009) 210-232. doi:10.1051/mmnp/20094309. URL http://www .mmnp-journal.org. docelec .univ-lyon1.fr/action/displayAbstract?

[22] A. Ducrot, V. Volpert, On a model of leukemia development with a spatial cell distribution, Mathematical Modelling of Natural Phenomena 2 (3) (2007) 101-120. doi:10.1051/mmnp:2007005.

URL http://www .mmnp-journal.org. docelec .univ-lyon1.fr/action/displayAbstract?

[23] S.-A. Gourley, J. Wu, Delayed non-local diffusive systems in biological invasion and disease spread, Nonlinear Dynamics and Evolution Equations, Amer. Math. Soc 48 (2006) 137-200. 
[24] J. Wu, Theory and Applications of Partial Functional Differential Equations, Springer, 1996. doi:10.1007/978-1-4612-4050-1.

URL http://www.springer.com/cn/book/9780387947716

[25] M. Adimy, A. Chekroun, B. Kazmierczak, Traveling waves in a coupled reaction-diffusion and difference model of hematopoiesis, Journal of Differential Equations 262 (7) (2017) 4085-4128. doi:10.1016/j.jde.2016.12.009.

URL http://www.sciencedirect.com/science/article/pii/S002203961630465X

[26] H. I. Freedman, P. Moson, Persistence definitions and their connections, Proceedings of the Americal Mathematical Society 109 (1990) 10251033. doi:10.2307/2048133.

URL http://www.jstor.org/stable/2048133

[27] H. Smith, Reduction of structured population models to threshold-type delay equations and functional differential equations: a case study, Mathematical Biosciences 113 (1) (1993) 1-23. doi:10.1016/00255564(93)90006-V.

URL http://www.sciencedirect.com/science/article/pii/002555649390006V

[28] J. W.-H. So, J. Wu, X. Zou, A reaction-diffusion model for a single species with age structure. I travelling wavefronts on unbounded domains, Proc. R. Soc. Lond. A 457 (2001) (2001) 1841-1853. doi:10.1098/rspa.2001.0789.

URL http: //rspa.royalsocietypublishing .org/content/457/2012/1841

[29] Z. Guo, Z.-C. Yang, X. Zou, Existence and uniqueness of positive solution to a non-local differential equation with homogeneous Dirichlet boundary condition - A non-monotone case, Communications on Pure and Applied Analysis 11 (5) (2012) 1825-1838. doi:10.3934/cpaa.2012.11.1825.

URL https : //www . aimsciences .org/journals/displayArticlesnew . jsp?paperID=7243

[30] M. H. Protter, H. F. Weinberger, Maximum Principles in Differential Equations, Springer-Verlag New York Inc., 1984.

[31] H. Amann, Fixed Point Equations and Nonlinear Eigenvalue Problems in Ordered Banach Spaces, SIAM Review 18 (4) (1976) 620-709. 
doi:10.1137/1018114.

URL http://epubs.siam.org/doi/10.1137/1018114

[32] C. V. Pao, Nonlinear Parabolic and Elliptic Equations, Springer Science \& Business Media, 1992.

URL https ://link. springer . com/book/10 . 1007\%2F978-1-4615-3034-3

[33] S. Bernard, L. Pujo-Menjouet, M. C. Mackey, Analysis of Cell Kinetics Using a Cell Division Marker: Mathematical Modeling of Experimental Data, Biophys J 84 (5) (2003) 3414-3424.

URL http://www.ncbi.nlm.nih.gov/pmc/articles/PMC1302899/ 